\title{
In vitro endoderm emergence and self-organisation in the absence of extraembryonic tissues and embryonic architecture
}

\author{
Stefano Vianello ${ }^{1 凶}$ and Matthias P. Lutolf ${ }^{1,2 凶}$ \\ ${ }^{1}$ Laboratory of Stem Cell Bioengineering, Institute of Bioengineering, School of Life Sciences (SV) and School of Engineering (STI), Ecole Polytechnique Fédérale de \\ Lausanne (EPFL), Lausanne, Switzerland \\ ${ }^{2}$ Institute of Chemical Sciences and Engineering, School of Basic Science (SB), EPFL, Lausanne, Switzerland
}

\begin{abstract}
The endoderm is the cell lineage which gives rise in the 44 embryo to the organs of the respiratory and gastrointestinal 45 system. In the mouse this may be the germ layer with the strongest association with its extraembryonic counterpart. Uniquely indeed, endodermal tissue does not just derive from descendants of the embryo proper (the epiblast) but instead arises from their gradual incorporation into an extraembryonic substrate (the visceral endoderm). Given the configuration of ${ }^{50}$ the early embryo, such a paradigm requires epiblast endoder- ${ }^{5}$ mal progenitors to negotiate embryonic compartments with ${ }^{52}$ very diverse epithelial character, a developmental contingency ${ }^{53}$ reflected by the fact that key early endodermal markers such 54 as Foxa2 and Sox17 have been consistently found to be em- 55 bedded within gene programmes involved in epithelialisation. 56
\end{abstract}

To explore the underlying cell biology of embryonic endoderm precursors, and to explore the relationship between endoderm development, epithelial identity, and extraembryonic mixing, we leveraged Gastruloids, in vitro models of early development. These self-organising three-dimensional aggregates of mouse embryonic stem cells do not possess an ${ }^{62}$ extraembryonic component, nor do they appear to display ${ }^{63}$ typical tissue architecture. Yet, they generate cells expressing 64 endodermal markers. By tracking these cells throughout in 65 vitro development, we highlight a persistent and uninterrupted 66 pairing between epithelial and endodermal identity, with 67 FoxA2+/Sox17+ endoderm progenitors never transitioning through mesenchymal intermediates and never leaving the epithelial compartment in which they arise. We also document the dramatic morphogenesis of these progenitors into a macroscopic epithelial primordium extending along the entire anterior-posterior axis of the Gastruloid, patterned into broad domains of gene expression. Corollarily we thus also highlight a strong epithelial component in Gastruloids, and thus the spontaneous emergence in vitro of stratified architectures and 75 germ layer compartmentalisation.

gastruloid | endoderm development | epithelium | self-organisation Correspondence: stefano.vianello@epfl.ch and matthias.lutolf@epfl.ch

\section{Introduction}

Nowotschin et al., 2019a), a "mucous layer" first described in chick embryos (Pander, 1817), and which we now know as "endoderm" (Allman, 1854; Oppenheimer, 1940).

In mouse, this "inner skin" actually first assembles on the outer surface of the embryo, through a unique choreography of cellular movements illustrated in Figure 1 (Burtscher \& Lickert, 2009; Kwon et al., 2008; Viotti et al., 2014). As the mouse embryo implants into the uterus of the mother, and extraembryonic tissues proliferate to impart to the conceptus its characteristic cylindrical shape (Smith, 1985), the mouse embryo (at the very tip of such cylinder) is little more than an epithelial mass of potent cells: the epiblast. Remarkably, such inconspicuous tissue will act as the origin of almost all cells of the developing embryo, and this by engaging a complex series of biological, mechanical, and topological transformations coupling proliferation to cell fate restriction and cell rearrangement. Such a complex series of transformations, gastrulation, is a key milestone of all embryonic development. As such, the initially multipotent and uncommitted cells of the early epiblast commit to specific fates, which are generally classified into the broad germ layer categories of ectoderm (skin and neural types), mesoderm (heart, muscles, and mesenchyme), and endoderm (internal organs, respiratory and digestive tract) (Arnold \& Robertson, 2009; Takaoka \& Hamada, 2012; Tam \& Behringer, 1997; Tam \& Loebel, 2007).

Yet we now know that at least one of these germ layers, the endoderm, does not come entirely from cells originating in the epiblast (Kwon et al., 2008; Nowotschin et al., 2019a,b; Viotti et al., 2014). Blurring the boundaries of prevalent developmental paradigms, and making such qualifiers somehow oxymoronic, "extraembryonic" cells (i.e. cells that segregated away from the epiblast early on even before implantation) also contribute to the embryo, and specifically to its endoderm-derived tissues. Indeed, the epiblast is not isolated from other tissues within the conceptus, and it is actually enveloped by a thin epithelium of so-called Visceral Endoderm (Figure 1A, in yellow). This latter sheet of cells on the surface of the epiblast is what one would classify as extraembryonic tissue, originating from much earlier lineage 


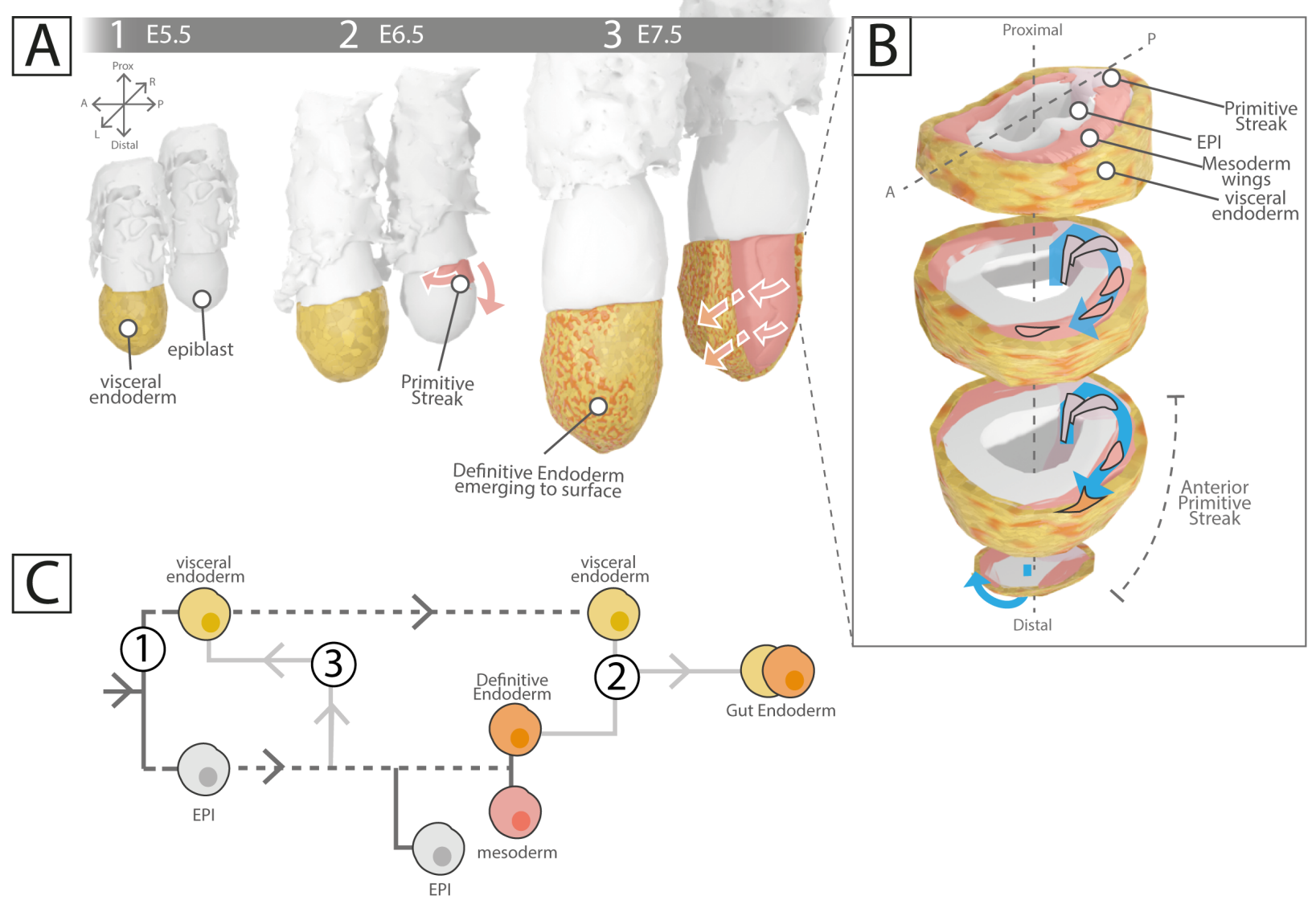

Fig. 1. Endoderm development in the mouse embryo. (A) Intercalation of (embryonic) definitive endoderm cells (orange) into the visceral endoderm epithelium (yellow, extraembryonic), during peri-gastrulation stages of mouse development. Embryos in the back row are represented with their visceral endoderm layer removed. In red, mesodermal cells emerging from the primitive streak and starting their posterior-to-anterior circumnavigation of the epiblast. (B) Exploded view of the mouse embryo at around E7.5, as if sectioned proximo-distally. At the posterior of the epiblast a zone of EMT (primitive streak, light purple) advancing towards the disal tip of the embryo mediates epiblast cell egression. Cells specified to mesoderm (red cells) leave the epiblast and form so-called mesodermal wings as they circumnavigate the epiblast. They are sandwiched between the epiblast they just left (gray), and the overlaying visceral endoderm (yellow). Defintive endoderm cells (orange) transit only shortly within the mesodermal compartment, and instead egress into the visceral endoderm. (C) The multiple origins of endodermal cells, as adapted from (Nowotschin et al., 2019b). 1) visceral endoderm cells, originating from earlier segregation between "embryonic" and "extraembryonic" cell types in the blastocyst, 2) intermixing with definitive endoderm cells arising from gastrulation (see previous panels), 3) direct delamination from the epiblast prior to gastrulation. $E=$ embryonic day.

segregation events (Chazaud et al., 2006). Originally thought 104 to be displaced away as the embryo develops, this thin sheet 105 of "extraembryonic" cells is the primary destination of a 106 subset of cells produced by the epiblast (i.e. embryonic ${ }_{107}$ cells, in orange throughout Figure 1A), that these embryonic ${ }_{108}$ cells are those that will make endoderm, and as such that the 109 visceral endoderm "extraembryonic" sheet in which these 110 cells intercalate becomes itself an integrated component of ${ }_{111}$ later embryonic structures (Burtscher \& Lickert, 2009; Kwon 112 et al., 2008; Nowotschin et al., 2019b; Viotti et al., 2014). ${ }_{113}$

What is the current model for how this process starts 115 and unfolds? At around embryonic day (E)6.25 asymmetric 116 signalling by extraembryonic tissues surrounding the epiblast ${ }_{117}$ break its symmetry (Stower \& Srinivas, 2018; Takaoka \& ${ }_{118}$ Hamada, 2012). One side of the epiblast starts becoming ${ }_{119}$ different from the rest (Figure 1A.2). At this side, which ${ }_{120}$ is defined as the posterior of the embryo and at which ${ }_{121}$ extraembryonic tissues concentrated high Wnt, BMP, and FGF signalling activity, epiblast cells respond by engaging so-called Epithelial to Mesenchymal Transition (EMT) programmes: they start losing attachment with the rest of the epithelium, they become motile and mesenchymal, they leave the epiblast (Arnold \& Robertson, 2009; Tam \& Behringer, 1997; Tam \& Loebel, 2007). Morphologically, the so-called Primitive Streak appears: a distally-expanding zone of EMT leading to delamination of epiblast cells and simultaneous commitment to embryonic fates (Hashimoto \& Nakatsuji, 1989; Williams et al., 2011). As epiblast cells undergo EMT and leave the epiblast, they start circumnavigating its outer surface, sandwiched under the overlaying visceral endoderm, forming wings of tissue converging towards the anterior of the embryo ((Hashimoto \& Nakatsuji, 1989; Saykali et al., 2019; Viotti et al., 2014); red intervening intervening tissue in Figure 1A.2 and 1A.3, and red cell trajectory in Figure 1B). These cells will generate mesodermal 
derivatives, heart and muscles (Tam \& Behringer, 1997). 179

Within the mesodermal compartment of the embryo 181 another cell type finds its way: endodermal cells. Like 182 mesodermal cells, these cells were once epiblast cells that ${ }_{183}$ left that epithelium to egress into the mesodermal compart- 184 ment (Burtscher \& Lickert, 2009). Rather than remaining 185 within these wings of mesoderm however, endodermal cells 186 start establishing contacts with the overlaying epithelium, 187 the visceral endoderm, into which they eventually integrate 188 (orange cell in Figure 11B; (Burtscher \& Lickert, 2009; 189 Kwon et al., 2008; Viotti et al., 2014)). The outer surface of 190 the embryo thus quickly becomes a mosaic of its original 191 resident population, that of visceral endoderm cells, and 192 of an increasing number of ingressing and intercalating ${ }_{193}$ endoderm cells of embryonic (epiblast) origin, so-called 194 Definitive Endoderm cells ((Burtscher \& Lickert, 2009; 195 Kwon et al., 2008; Viotti et al., 2014); orange cells in 196 Figure 1A.3). This sheet of cells will later form pockets 197 at the anterior and posterior of the embryo, and finally 198 fold along its midline to close into a tube that will end up 199 internalised within the embryo (not illustrated; (Carlson, 200 2014; Lewis \& Tam, 2006; McGrath \& Wells, 2015)). The 201 gut tube has formed, and along its entire length progeni- 202 tors of all endoderm-derived visceral organs will emerge 203 and take shape (Carlson, 2014; McGrath \& Wells, 2015). 204 refer to as "gut endoderm", i.e. the endodermal sheet which 207 folds and closes to give rise to the embryonic gut tube, is 208 thus actually a mixture of cells of very different origins, even 209 though these converge towards similar (yet not identical) 210 endpoint molecular signatures (Nowotschin et al., 2019a,b; 211 Viotti et al., 2014). The multiple contributions to gut 212 endoderm described above are summarised in Figure 1C (as ${ }_{213}$ adapted from e.g. (Nowotschin et al., 2019a)). In addition ${ }_{214}$ to the first contribution of Visceral Endoderm cells by early ${ }_{215}$ segregation within the inner cell mass of the early embryo 216 (Figure 1C.1), and to the later intercalation of Defintive ${ }_{217}$ Endodem cells (Figure 1C.2), we also highlight a third ${ }_{218}$ source of cells: epiblast cells bypassing EMT and altogether ${ }_{219}$ bypassing transit within the mesodermal compartment of the 220 embryo. These cells leave the epiblast to directly intercalate 221 into the visceral endoderm, a contribution that has been 222 documented to occur at the distal tip of the pre-gastrulation 223 mouse embryo, zone of maximal mechanical stress (Hira- 224 matsu et al., 2013; Matsuo \& Hiramatsu, 2017), and that 225 has found support from single-cell transcriptome analyses 226 (Nowotschin et al., 2019b). Direct epiblast to endoderm 227 transitions are particularly interesting, as even endodermal ${ }_{228}$ progenitors that do classically egress from the epiblast 229 into the mesodermal space might do so by EMT processes 230 different than those governing the egression and specification ${ }_{231}$ of mesoderm (Bardot \& Hadjantonakis, 2020; Burtscher \& 232 Lickert, 2009), and the issue remains contentious. Currently, 233 data suggests that egressing endodermal progenitors do 234 not completely lose their epithelial character but instead ${ }_{235}$ transiently redistribute their surface adhesion molecules as they travel along the mesodermal compartment, until they contact their new epithelial niche, the visceral endoderm, and fully repolarise (Bardot \& Hadjantonakis, 2020; Kwon et al., 2008; Nowotschin et al., 2019a; Viotti et al., 2014).

Uncertainty remains regarding many of the steps described above, and on the exact nature of the transition states that embryonic endoderm precursors traverse as they leave epiblast potency and refine endodermal identity (Bardot \& Hadjantonakis, 2020; Ferrer-Vaquer et al., 2010; Lewis \& Tam, 2006). Notably, evidence for so-called "mesendodermal progenitors", whereby bipotential cells able to give rise to both mesoderm and endoderm would exist within or outside the epiblast, is debated in mouse (Lewis \& Tam, 2006) despite the clear existence of such progenitor state in other developmental models (e.g. sea urchin and roundworms; (Peter \& Davidson, 2010; Sulston et al., 1983)). Certainly, segregation between the two germ layers in the mouse is documented already at very early stages, and actually within the very pre-streak epiblast (Burtscher \& Lickert, 2009). Uncertainty also remains on whether endodermal cells egress from the epiblast through mechanisms common to those of egressing mesodermal cells or through alternative mechanisms. Crucially, the observation that endodermal cells can be seen to have left the epiblast in regions which the primitive streak has not yet reached (Burtscher \& Lickert, 2009), and that those within mesoderm wings of the embryo have not lost their epithelial identity (Viotti et al., 2014) raise interest in the relationship between epitheliality and endodermal identity (Ferrer-Vaquer et al., 2010; Nowotschin et al., 2019a; Viotti et al., 2014). Given the recent spotlight on the mixed composition and distribution of gut endoderm cells (Nowotschin et al., 2019b), one also wonders whether extraembryonic and embryonic endoderm cells play distinct essential roles within this primordium and its derivatives. In embryos where embryonic endoderm precursors cannot integrate their extraembryonic substrate and remain trapped within the mesodermal compartment, these seem to lose their identity and embryos do not form midgut and hindgut (Kanai-Azuma et al., 2002; Viotti et al., 2014).

As a platform to explore the underlying cell biology of embryonic endoderm precursors, and to explore the relationship between endoderm development, epithelial identity, and extraembryonic mixing, we use Gastruloids (Beccari et al., 2018; Turner et al., 2017; van den Brink et al., 2014). These stem cell aggregates develop in vitro in times and patterns that are surprisingly but consistently reminiscent of in vivo embryonic development. While mainly characterised in terms of mesodermal and neuromesodermal development (van den Brink et al., 2020), they have been crucially also found to specify endodermal identities ((Beccari et al., 2018; Cermola et al., 2019; Pour et al., 2019; Turner et al., 2017; van den Brink et al., 2014; Veenvliet et al., 2020); see also Discussion). Work with this system reflects a paradigm whereby leaving cells to their own self-organisation ex- 
poses intrinsic cellular programmes and developmental 290 modules that would be otherwise masked by the regula- 291 tive context of normal embryonic development (Davies, 292 2017; Shahbazi \& Zernicka-Goetz, 2018; Turner et al., 293 2016). In this perspective, the absence of typical embryonic 294 architecture, compartmentalisation, and extraembryonic 295 tissues, makes Gastruloids particularly suitable to study 296 the relevance of these features to endoderm development. 297

We here highlight a persistent and uninterrupted pair- 299 ing between epithelial and endodermal identity, with 300 FoxA2+/Sox17+ endoderm progenitors never transitioning ${ }_{30}$ through mesenchymal intermediates and never leaving 302 the epithelial compartment in which they arise. We also 303 document the dramatic morphogenesis of these progenitors 304 into a macroscopic epithelial primordium extending along ${ }_{305}$ the entire anterior-posterior axis of the Gastruloid, patterned ${ }_{300}$ into broad domains of gene expression. Corollarily we thus 307 also highlight a strong epithelial component in Gastruloids, 308 and thus the spontaneous emergence in vitro of stratified 309 architectures and germ layer compartmentalisation.

\section{Results}

To investigate the emergence, dynamics, and patterning of ${ }_{314}$ endoderm progenitors in vitro we started by generating Gas- ${ }_{315}$ truloids (Baillie-Johnson et al., 2015; Beccari et al., 2018; ${ }_{316}$ van den Brink et al., 2014). Accordingly, we aggregated $300_{317}$ mouse embryonic stem cells of a TBra/Sox 1 double reporter ${ }_{318}$ line (described in (Deluz et al., 2016)) whose output Gastru- ${ }_{319}$ loids have been extensively characterised in published litera- ${ }_{320}$ ture and for which we have documented expression of mark- ${ }_{221}$ ers of all three germ layers (Beccari et al., 2018); see Ma- ${ }_{222}$ terials \& Methods). As expected, when 300 of these mouse ${ }_{323}$ embryonic stem cells are seeded in individual wells of a low- ${ }_{324}$ adhesion 96well plate and maintained in N2B27 medium, ${ }_{325}$ these sediment to the bottom of the well and aggregate to- ${ }_{326}$ gether in the first $48 \mathrm{~h}$ of culture to form a compact sphere ${ }_{327}$ with defined edges by $72 \mathrm{~h}$ (Figure 2). A pulse of the glyco- ${ }_{328}$ gen synthase kinase (GSK) 3 inhibitor (CHIR99021, Chiron) ${ }_{329}$ is then applied as a trigger of "gastrulation" and as to mimic ${ }_{3 з 0}$ the increase in Wnt signalling experienced by cells of the ${ }_{331}$ posterior mouse epiblast. Accordingly, the aggregate breaks ${ }_{332}$ symmetry (Figure 2A, asterisk). Morphologically, the spherical $72 \mathrm{~h}$ aggregate becomes oblong by $96 \mathrm{~h}$, and extends $\mathrm{a}_{334}$ long protrusion that grows over time (120h, 144h). This pos- ${ }_{335}$ terior protrusion is marked by TBra (Brachyury) expression, ${ }_{336}$ marker of the posterior primitive streak and of the embryonic ${ }_{337}$ tail bud, and found to similarly define the posterior of the ${ }_{338}$ Gastruloid (Beccari et al., 2018; Turner et al., 2017; van den ${ }_{3 з}$ Brink et al., 2014).

\section{Emergence and patterning of endodermal markers.}

Emergence of early endodermal markers. In the embryo, precursors of the definitive endoderm appear to be found within ${ }_{345}$ epiblast cells marked by expression of the transcription factor FOXA2 (Burtscher \& Lickert, 2009). These FOXA2+ cells would be initially intermingled with TBra+ (TBXT) cells at the proximal posterior side of the embryo, and then resolve as a homogeneous FOXA2+ population marking the distal portion of the epiblast, and thus the epiblast region anterior to the leading edge of the primitive streak (Bardot et al., 2017; Burtscher \& Lickert, 2009). While cells in the intervening epiblast region and co-expressing FoxA2 and TBra may be progenitors of cardiac mesoderm types (Bardot et al., 2017), FOXA2+ cells of the distal epiblast are posited to leave the columnar epithelium, upregulate FoxA2, and move within the wings of mesoderm enveloping the epiblast ((Burtscher \& Lickert, 2009; Kwon et al., 2008; Viotti et al., 2014), see also Figure 1B). FOXA2+ cells that contact the overlaying visceral endoderm would upregulate Sox17 (Viotti et al., 2014), leave the mesodermal domain, integrate within this new epithelium, and join the cohort of cells that will eventually form the gut endoderm (Burtscher \& Lickert, 2009; Kwon et al., 2008; Viotti et al., 2014). Given the relevance of FoxA2 and Sox17 for endoderm development (Dufort et al., 1998; Kanai-Azuma et al., 2002; Monaghan et al., 1993; Sasaki \& Hogan, 1993), and their prevalent use in the gastruloid/embryoid literature as early endoderm markers (Beccari et al., 2018; Pour et al., 2019; Turner et al., 2017; van den Brink et al., 2014; Veenvliet et al., 2020), we decided to track their emergence and patterns of expression at the earliest stages of Gastruloid development.

At $72 \mathrm{~h}$, when the Gastruloid is still spherical and has just received the CHIR stimulus that will drive it through differentiation and morphogenesis, FOXA2+ cells can be seen scattered throughout the aggregate, intermingled with TBra+ cells (Figure 2B.1, 72h). Just 24h later ( $\mathrm{t}=96 \mathrm{~h})$, and as TBra+ cells resolve into a pole that will accordingly define the posterior of the Gastruloid (van den Brink et al., 2014), FOXA2+ cells form clusters and segregate away from the TBra+ pole along the newly defined axis of the aggregate (Figure 2B.1, 96h) . While few FOXA2+/TBra+ (double-positive) cells can be distinguished at this stage, most cells are either TBra+ at the posterior of the Gastruloid, or FOXA2+ as scattered clusters along the anterior (Figure 2B.1,bottom).

In contrast to TBra and FOXA2, SOX17 cannot be detected at $\mathrm{t}=72 \mathrm{~h}$, but only starts appearing later (Figure 2B.2). At $\mathrm{t}=96 \mathrm{~h}$, scattered unclustered SOX17+ cells appear within the elongating Gastruloid, and co-staining for FOXA2 shows these cells as representing a nearly exclusive subset of the FOXA2+ population (Figure 2B.3). We thus observe, at the earliest timepoints of Gastruloid response to CHIR, ordered emergence of key endodermal markers in sequence and patterns that are consistent with what is observed in the embryo. Not only SOX17+ cells emerge later and within a population of FOXA2+ cells (as seen in the embryo, (Viotti et al., 2014)), these cells sort from an initially TBra-intermingled population to later define posterior and anterior domains along the AP axis of the Gastruloid, just as is observed in the epiblast of the early primitive streak embryo (Burtscher \& Lickert, 2009). 
bioRxiv preprint doi: https://doi.org/10.1101/2020.06.07.138883; this version posted June 10, 2020. The copyright holder for this preprint (which was not certified by peer review) is the author/funder, who has granted bioRxiv a license to display the preprint in perpetuity. It is made available under aCC-BY 4.0 International license.
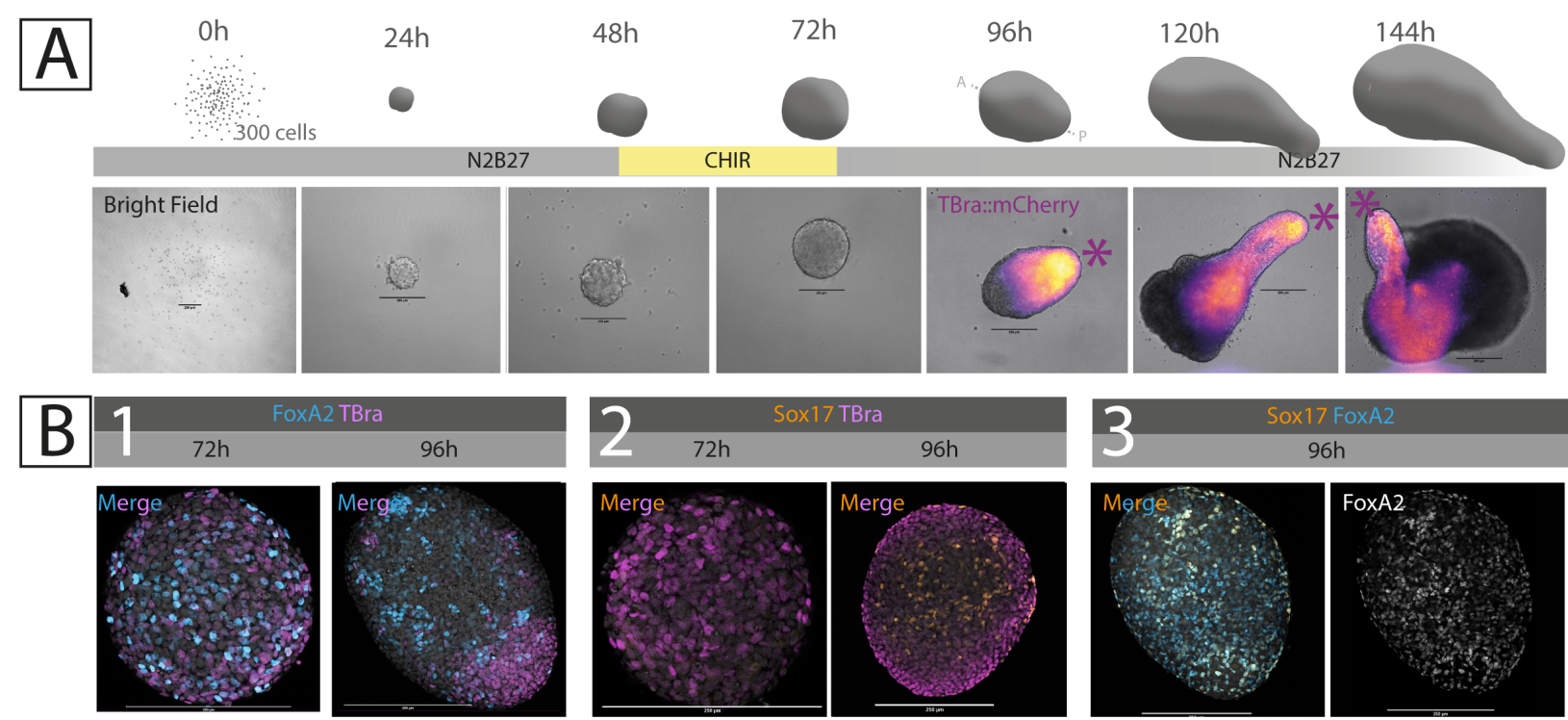
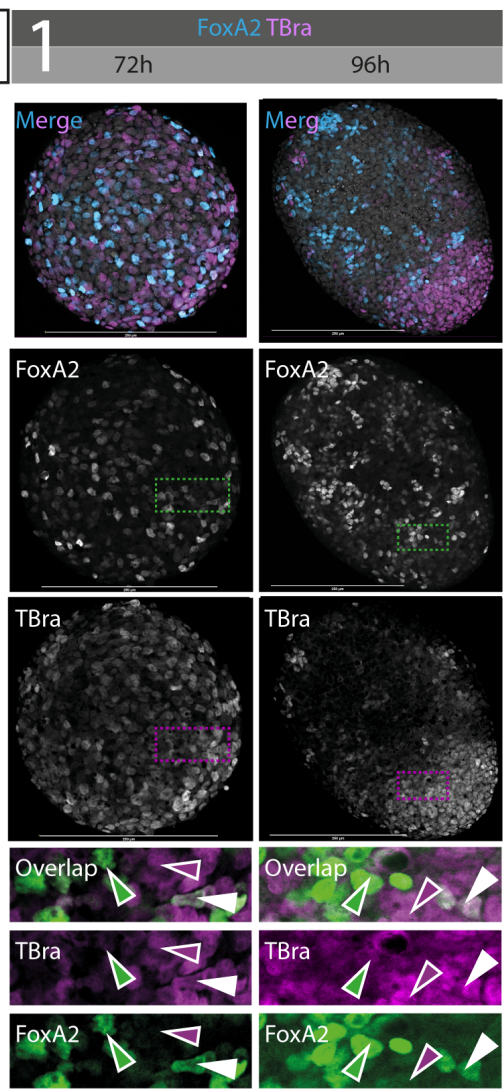
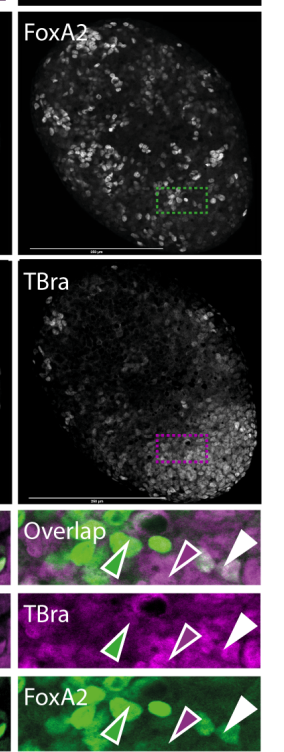
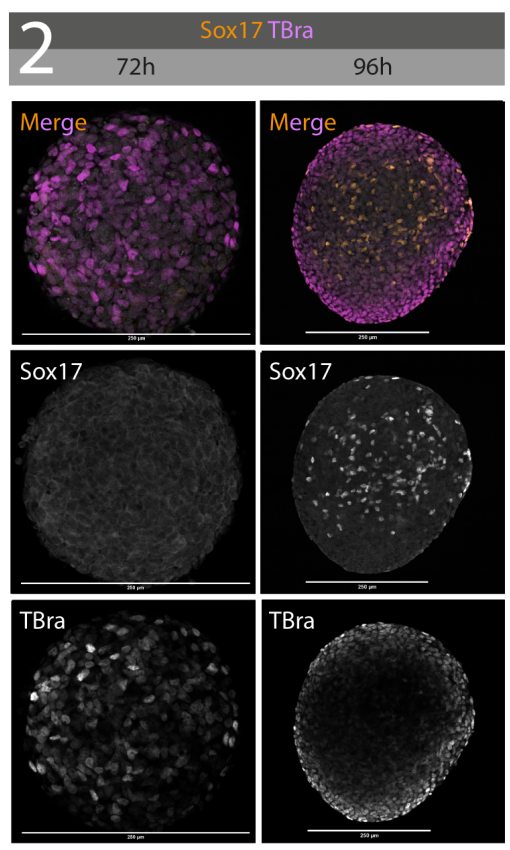
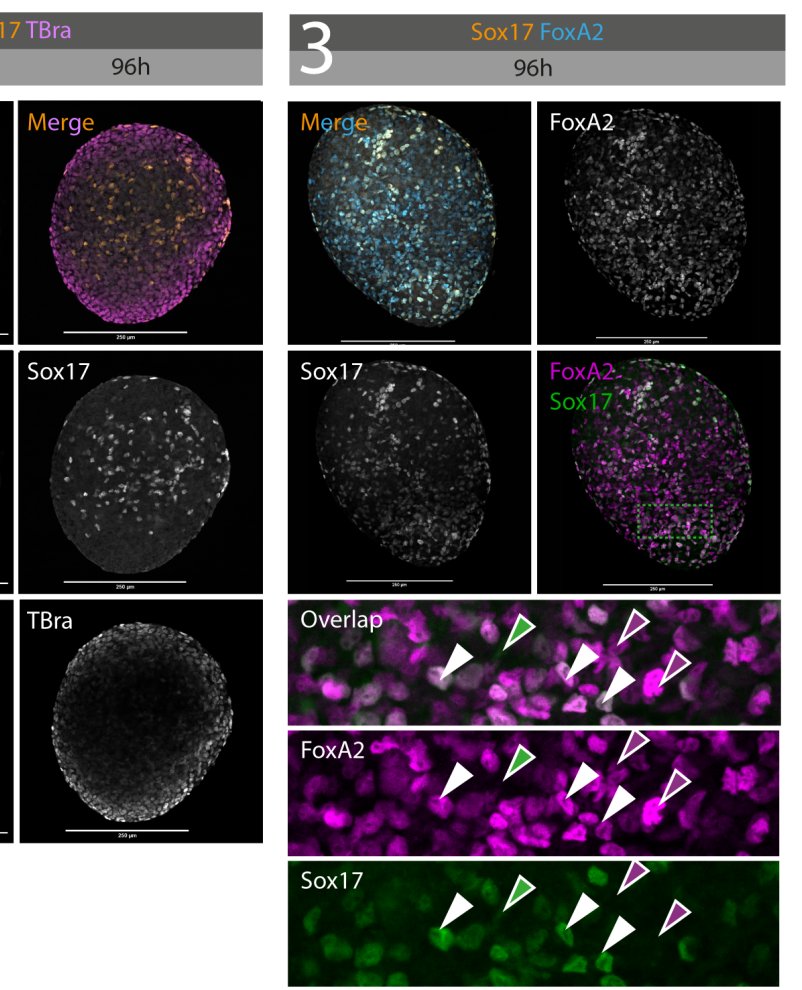

Fig. 2. Emergence and patterning of endodermal markers in Gastruloids. (A) Summary schematic of the Gastruloid generation protocol (see Methods), and brightfield

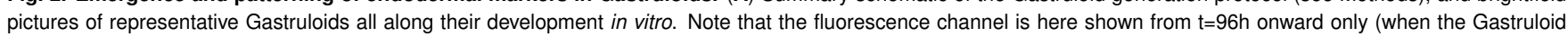
becomes polarised). Reporter expression starts at $t=72 h$, homogeneously throughout the spheroid (see (Turner et al., 2017)). (B) Immunostaining against the posterior epiblast and primitive streak marker TBra, and classical endodermal progenitor markers FoxA2 and Sox17, at 72h and 96h of Gastruloid development. SOX17+ cells appear one day later TBra+ and FOXA2+ cells, and are a nearly exclusive subset of the latter. Marker colocalisation is shown in green and magenta, with double-positive cells appearing white (examples of single-positive and double-positive cells highlighted by single-colour and white arrowheads respectively). Scale bar is always 250 um. Asterisk indicates the posterior of the Gastruloid (based on TBra expression).

Cellular biology of endodermal cells. In vivo, FoxA2+ (and 361 thus Sox17+) cells are expected to occupy and traverse very 362 different embryonic compartments throughout their journey. 363 As such, FOXA2+ cells would first emerge within the ${ }_{364}$ columnar epithelial tissue of the epiblast, they would then 365 egress and mix with the mesenchymal mesodermal cell types 366 circumnavigating the embryo as mesodermal wings, and 367 they would finally re-integrate the epithelium on the surface 368 of the embryo ((Kwon et al., 2008; Viotti et al., 2014), as ${ }_{369}$ illustrated in Figure 1B). Sox17 expression appears to be 370 even more intimately associated with transitions between 371 compartments, and has been reported to be expressed once 372 endodermal precursors contact and integrate within the 373 surface epithelium (Viotti et al., 2014). We thus sought to 374 resolve whether cells expressing endodermal markers in Gastruloids were equivalently moving across compartments, with particular attention to their epithelial identity. Indeed, we observe SOX17+ cells in the absence of surface epithelial layer on which these would eventually integrate in vivo.

Co-staining for the the epithelial marker CDH1 (Ecadherin, adherens junction) shows that both TBra+ and FOXA2+ cells specified within the $t=72 \mathrm{~h}$ Gastruloid are emerging within a cellular aggregate that is uniformly epithelial (or, at least, epithelioid since epithelial architecture is missing, Figure 3A), consistently with the epithelial context of the epiblast of the early gastrulation embryo in which TBRA+ and FOXA2+ cells have been described 
bioRxiv preprint doi: https://doi.org/10.1101/2020.06.07.138883; this version posted June 10, 2020. The copyright holder for this preprint (which was not certified by peer review) is the author/funder, who has granted bioRxiv a license to display the preprint in perpetuity. It is made available under aCC-BY 4.0 International license.
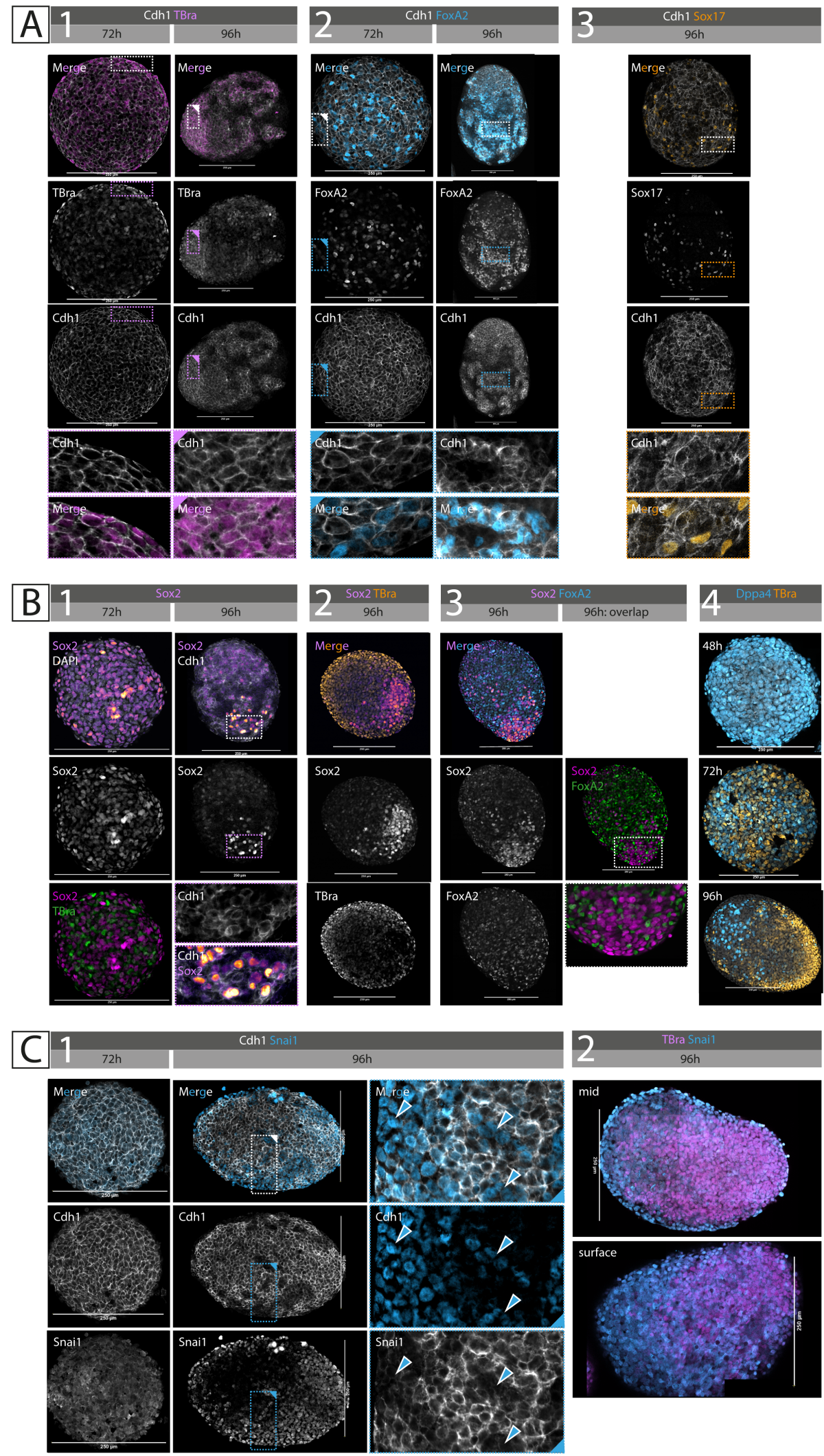

Fig. 3. Endoderm progenitors are epithelial in nature, reside within an epiblast-like compartment, and are spared by classical EMT. (A) Immunostaining against the epithelial molecule E-cadherin (Cdh1) at $72 \mathrm{~h}$ and $96 \mathrm{~h}$ of Gastruloid development. Notice the drastic fragmentation of epithelial integrity between the two timepoints. Co-stained, are the posterior epiblast and primitive streak marker TBra, and classical endodermal progenitor markers FoxA2 and Sox17. Cells expressing either marker are consistently also $\mathrm{CDH} 1+$. (B) Immunostaining against the epiblast marker Sox2 shows its segregation at the anterior of the 96h Gastruloid, thus defining a separate domain from FoxA2+ cells. A similar pattern is highlighted by the pluripotency marker Dppa4. (C) Immunostaining against the classic EMT regulator Snail-1 (Snai1) shows patterns of expression complementary to those of CDH1. Sna1-mediated EMT is widespread throughout the 96h Gastruloid, and marks cells enveloping the aggregate. Scale bar is always 250um. Marker colocalisation is shown in green and magenta, with double-positive cells appearing white (examples of single-positive and double-positive cells highlighted by single-colour and white arrowheads respectively) 
to first emerge (Burtscher \& Lickert, 2009; Lee et al., 432 2007). Cells of the $t=72 \mathrm{~h}$ Gastruloid all show homoge- 433 neous membrane CDH1 localisation, as likely expected ${ }_{434}$ for an aggregate of embryonic stem cells transitioning to- 435 wards EpiSC states (Hamidi et al., 2020; Turner et al., 2017). 436

Interestingly, the CDH1 landscape of the $t=96 \mathrm{~h}$ Gastru- 438 loid, one day later, is radically different: as Gastruloids ${ }_{439}$ respond to the CHIR pulse, CDH1 expression becomes 440 patchy. The original $\mathrm{CDH} 1$ continuum of the $72 \mathrm{~h}$ spherical ${ }_{441}$ Gastruloid displays signs of clear fragmentation by $t=96 h_{442}$ (Figure 3A). At the posterior, $\mathrm{CDH} 1+$ cells remain clustered ${ }_{443}$ and maintain expression of TBra in a configuration analogous 444 to that of the epiblast of the posterior or incipient primitive 445 streak ((Burtscher \& Lickert, 2009; Herrmann, 1991), Figure 446 3A.1), while anteriorly CDH1 continuity is increasingly 447 interrupted by intervening non-epithelial cells (interpreted ${ }_{448}$ to be mesoderm). Very interestingly, all FOXA2+ cells seen 449 at this stage are contained within this disaggregating $\mathrm{CDH} 1450$ core, just as the newly specified SOX17+ cells emerging ${ }_{451}$ within such FOXA2+ population (Figure 3A.2 and Figure 452 3A.3). We never observe FOXA2+ or (FOXA2+/)SOX17+ ${ }_{453}$ cells outside of the perimeter defined by the $\mathrm{CDH} 1+$ islands. 454

These findings are particularly significant in that one 456 might naively expect to observe FOXA2+ cells to be leaving ${ }_{457}$ their CDH1+ ("epiblast") substrate and for FOXA2+ and FOXA2+/SOX17+ cells to be found in the emerging mes- ${ }^{458}$ enchymal compartment. Significantly however, FOXA2+ 459 cells leaving the epiblast in vivo do not lose CDH1 expres- ${ }^{-40}$ sion either, but rather relocalise it isotropically until they ${ }^{461}$ make contact with and reintegrate the overlaying visceral ${ }^{462}$ endoderm (Viotti et al., 2014). The isotropic CDH1 starting ${ }^{463}$ point of the Gastruloid at $\mathrm{t}=72 \mathrm{~h}$ might thus reconcile in ${ }^{464}$ vitro and in vivo findings by explaining the retention of 465 SOX17+ cells in the original "epiblast" compartment. Cer- ${ }^{466}$ tainly, the observation of such pervasive CDH1 expression ${ }^{467}$ within Gastruloids, at least those derived from the stem cell ${ }^{468}$ line used in this study (see Discussion), challenged our pre- 469 conception of Gastruloids as mainly mesenchymal organoids. ${ }^{470}$

To test the underlying identity of the Cdh1 domain of $t=72 h{ }^{472}$ and $\mathrm{t}=96 \mathrm{~h}$ Gastruloids, and to check whether endodermal ${ }^{473}$ identities were indeed emerging and remaining associated 474 with an "epiblast"-like domain, we co-stained Gastruloids for 475 pluripotency and epiblast markers (Figure 3B). Staining for ${ }^{476}$ SOX 2 shows that the $t=72 \mathrm{~h}$ spheroid is indeed a collection ${ }^{477}$ of SOX2+ cells (maintained from earlier timepoints, see data 478 in (Beccari et al., 2018)), and newly emerging TBra+ cells ${ }^{479}$ (Figure 3B.1). As such the emerging scattered FOXA2+ 480 population at this stage (also intermingled with TBra+ cells) ${ }^{481}$ is likely emerging on this very $\mathrm{CDH} 1+/ \mathrm{SOX} 2+$ substrate. ${ }^{482}$ Yet, as gastruloid "gastrulation" progresses, the fragmenting ${ }^{483}$ epithelial compartment only maintains high SOX2 at a pole ${ }^{484}$ (opposite to the TBra pole, i.e. at the anterior, Figure 3B.2), ${ }^{485}$ such that the rest of the epithelium, where FOXA2+ and ${ }^{486}$ TBra+ identities segregate, shows low or no SOX2 (Figure ${ }^{487}$
3B.3). A similar pattern of segregation and maintenance of potency at the anterior tip of the $t=96 \mathrm{~h}$ gastruloid is highlighted by the observed dynamics of the pluripotency marker DPPA4, marking the entire stem cell aggregate at $\mathrm{t}=48 \mathrm{~h}$, segregating from emerging TBra+ cells at $\mathrm{t}=72 \mathrm{~h}$, and being restricted at the anterior by $\mathrm{t}=96 \mathrm{~h}$ (Figure 3B.4).

Our observations are consistent with a model where endodermal identities differentiate without ever leaving the epiblast compartment, or at least where endodermal precursors retain $\mathrm{CDH} 1$ expression throughout their development. Indeed, the $\mathrm{CDH} 1+$ mass of the $\mathrm{t}=96 \mathrm{~h}$ Gastruloid may itself represent different embryonic compartments: one of epiblast, maintaining potency markers at the anterior and downregulating SOX2 at the posterior where FOXA2+ and TBra+ cells segregate to define respectively distal and proximal posterior epiblast identities, and one of FOXA2+/SOX17+ endodermal precursors that would normally be found in the mesenchymal compartment of the embryo but here remain attached to the epiblast given the isotropic CDH1 expression of all other compartments. In either case, we strengthen the case for direct epiblast-to-endoderm transitions that may not require classic EMT or transitions through so-called mesendodermal intermediates (Ferrer-Vaquer et al., 2010; Kubo et al., 2004; Lewis \& Tam, 2006; Pfendler et al., 2005; Tada et al., 2005).

Gastruloids undergo widespread EMT, which spares endodermal precursors. To test whether the observed fragmentation of the epithelial core of the Gastruloid is consistent with EMT-like processes one would expect for an in vitro model of gastrulation, and how this relates to the apparent failed EMT of endodermal precursors in the Gastruloid, we performed immunostaining for the EMT master regulator Snail (SNAI1) ((Cano et al., 2000; Carver et al., 2001), Figure 3C).

While SNAI1 is only detected at low levels in the cytoplasm of cells of $\mathrm{t}=72 \mathrm{~h}$ Gastruloids (all $\mathrm{CDH} 1$ positive, as shown before), large swathes of cells with strong nuclear SNAI1 signal are observed at $\mathrm{t}=96 \mathrm{~h}$ (Figure 3C.1). Crucially, these patches of SNAI1+ cells are consistently observed to mark the cells intervening between fragments of the CDH1 core. Optical cross-sections at the midplane of $\mathrm{t}=96 \mathrm{~h}$ Gastruloids show SNAI1+ cells forming an envelope at the surface, and establishing a posterior to anterior gradient in continuity with the TBra+ posterior (Figure 3C.2).

We thus notice that Gastruloid "EMT" seems to be a generalised rather than localised process, originating at several points within the CDH1+ "epiblast" substrate. Alternatively, SNAI1+ mesodermal types originating from a localised EMT origin may be migrating and physically displacing $\mathrm{CDH} 1+$ cells, leading to the observed fragmented appearance. Regardless, the retention of FOXA2+ cells within $\mathrm{CDH} 1+$ islands, in an environment of widespread EMT, seems to suggest that these cells are not leaving the epiblast and are either transitioning through endodermal differentiation within their original epiblast-like environment 
or attempting to leave the epithelium by Snail-independent 545 mechanisms (Bardot \& Hadjantonakis, 2020) and either ${ }_{546}$ rapidly reintegrating it at short timescales or remaining ${ }_{547}$ attached to it through homotypic $\mathrm{CDH} 1$ interactions. 548

\section{Formation of an endoderm-like primordium.}

The epithelial core of the Gastruloids undergoes dramatic ${ }_{554}$ architectural rearrangements. To evaluate the later fate of ${ }_{555}$ endodermal cells within the gastruloid core, we further ${ }_{556}$ tracked Cdh1, FoxA2, and Sox17 patterns of expression as the Gastruloid undergoes morphogenesis and elonga- 557 tion from $\mathrm{t}=96 \mathrm{~h}$ onward ((Beccari et al., 2018), Figure 4). 558

Strikingly, the fragmented CDH1+ core of the $t=96 \mathrm{~h}{ }_{560}$ Gastruloid gradually re-organises in complex and stream- ${ }_{56}$ lined elongated architectures extending along the entire 562 length of the Gastruloid (Figure 4A). Over time, the ${ }_{563}$ tear-drop shaped, fragmented $\mathrm{CDH} 1+$ core of the $t=96 \mathrm{~h} 564$ Gastruloid tapers into a multi-branched, whisk-shaped 565 epithelial primordium $(\mathrm{t}=120 \mathrm{~h}, 144 \mathrm{~h})$, which in turn resolves 566 in a single rod-like tissue that follows the outer geometry ${ }_{567}$ of the Gastruloid (144h onward). This epithelial structure, 568 consistently seen in all $(n=97 / 99$ imaged Gastruloids, N 569 $=6$ independent experiments) samples (Figure 5), emerges 570 on the surface of the Gastruloid at the very posterior (and 571 is indeed an extension of it), sometimes displaying multiple 572 surface points in the region (white arrowhead Figure 4A, 573 right), and occasionally also resurfaces at the anterior at 574 much later timepoints $(\mathrm{t}=168 \mathrm{~h})$. Crucially, cells of this 575 epithelial core are marked by FoxA2 at all stages of de- 576 velopment (Figure 4A, bottom row). Macroscopically, this 57 epithelial mass is already distinguishable in brightfield as 578 a rod-like structure of compact cells extending from the 579 posterior and gradually becoming enveloped by anteriorly- 580 extending wings of loser, mesenchymal-like cells (Figure 581 4B.1). Co-staining of CDH1 with the pan-mesodermal 582 marker FoxC1 (Sasaki \& Hogan, 1993) clearly identifies 583 the enveloping tissue as mesodermal (whose differentiation 584 will give rise to the variety of trunk and cardiac structures 585 described in (Rossi et al., 2019; van den Brink et al., 586 2020; Veenvliet et al., 2020)) and highlights a multilayered 587 architectural organisation of the Gastruloid model, with 588 interfacing epithelial and mesenchymal tissue (Figure 589 4B.2). Interestingly, such coupled configuration raises the 590 possibility that the two compartments may engage in pro- 591 ductive developmental interaction, possibly stimulating the 592 development of cells types that would not otherwise emerge ${ }_{593}$ in either alone (as described in vivo e.g. in (Han et al., 2019)). 594

Interestingly, the CDH1+ primordium appears to un-596 dergo epithelial maturation over time. Whereas $\mathrm{CDH} 1+{ }_{597}$ cells of the $\mathrm{t}=72 \mathrm{~h}$ Gastruloid represented more an epithelioid 598 state, with expression of epithelial markers but without 599 epithelial architecture, the $\mathrm{t}=120 \mathrm{~h}$ and onward CDH1 mass 600 shows signs of apico-basal polarity with polarised arrange- 601 ment of GM130 (Figure 4B.3), and gradual deposition of discontinuous stretches of laminin (LAMA1 subunit) at the interface with the overlaying mesoderm (Figure 4B.4). We could not prove the existence of a continuous lumen within this epithelium, suggesting this structure to be more akin to a plastic epithelial mass rather than a defined continuous tube (data not shown). When present, supernumerary points of contact with the posterior surface of the Gastruloid do however seem to be consistently associated with rosetting and local invagination (see Figure 4A), even though we cannot at this point demonstrate continuity of these cavities with the rest of the $\mathrm{CDH} 1$ core.

Endodermal patterning along the AP axis of the Gastruloid. Having observed such dramatic and unexpected epithelial rearrangements over late Gastruloid development, and such tight association between this newly formed $\mathrm{CDH} 1$ core and the endodermal marker FOXA2 (Figure 4A and 5C), we proceeded to further characterise the identity of these cells and their possible patterning along the AP axis (Figure 4C, indeed observed for cells of the overlaying mesodermal compartment (Beccari et al., 2018; van den Brink et al., 2020)).

Matching the surprising increase in the number of $\mathrm{CDH} 1+/ \mathrm{FOXA} 2+$ cells from $\mathrm{t}=96 \mathrm{~h}$ to $\mathrm{t}=120 \mathrm{~h}$, where FOXA2 seems to mark almost the entirety of the CDH1+ primordium, SOX17 immunostaining also reveals a surprising increase in SOX17+ numbers, with cells extending from the neck of the CDH1 primordium (and often from the "hole" like surface openings described above) up to anteriormost extremity of the epithelium (Figure 4C.2 and Figure 4C.3). Immunostaining for the posterior primitive streak and tailbud marker TBra (Figure 4C.1) reveals that while FOXA2+ and SOX17+ cells may well be in continuity with the CDH1+/TBRA+ Gastruloid tip, they organise themselves just anterior to it. Batch quantification of signal intensity along the AP axis of Gastruloids (see plot in Figure 4C) indeed highlights reproducible patterning where the posterior 5th of the CDH1 primordium is marked by TBra+/CDH1+/FOXA2- cells, while the rest is populated by TBra-/CDH1+/FOXA2+ (and SOX17+) cells, an epithelialised endoderm whose continuity with the TBrat pole might be explained by persistent homotypic interaction between different $\mathrm{CDH} 1+$ tissues. Of note, few TBra+ cells do seem to also extend deeper into the FOXA2+ domain (Figure 4C.5), and constitute a CDH1+/TBra+/FOXA2+ population that may be consistent with midline embryonic structures (Burtscher \& Lickert, 2009; Yamanaka et al., 2007).

At the very posterior of the Gastruloid (and thus of the CDH1 primordium), the posterior marker CDX2 (Beck et al., 1995) marks not only the TBra+/CDH1+ cells of the gastruloid tip, and CDH1- mesenchymal cells emerging laterally from it (Figure 4C.4), but also $(\mathrm{CDH} 1+/) \mathrm{SOX} 17+$ cells at the posterior limit of the SOX17+ domain (Figure 4C.7). Comparison of these structures with the caudal intestinal portal forming during in vivo endoderm development (Beccari et al., 2018) are enticing but will need to wait 
bioRxiv preprint doi: https://doi.org/10.1101/2020.06.07.138883; this version posted June 10, 2020. The copyright holder for this preprint (which was not certified by peer review) is the author/funder, who has granted bioRxiv a license to display the preprint in perpetuity. It is made available under aCC-BY 4.0 International license.
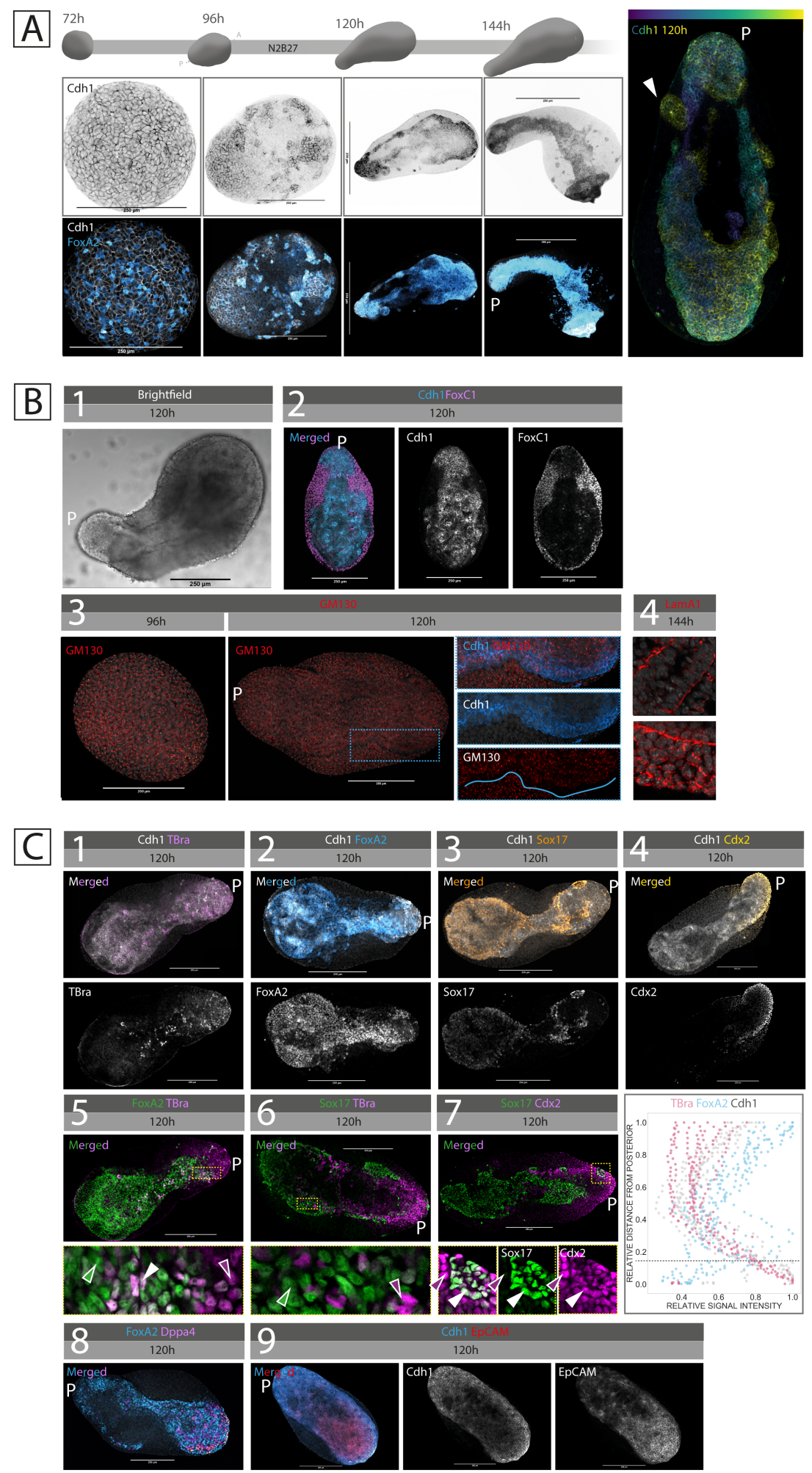

Fig. 4. An epithelial primordium forms at the core of elongating Gastruloids, and shows patterned expression of endodermal markers. (A) Representative immunostainings of Gastruloids undergoing elongation and morphogenesis. CDH1+/FOXA2+ cells appear to segregate at the core of the Gastruloid and form an elongated primordium spanning the entire length of the aggregate. On the right, depth-coded projection of the primordium in a 120h Gastruloid. White arrowhead highlights a posterior lateral opening to the surface. (B) Macroscopic appearance of the epithelial primordium in its relationship with the overlaying mesodermal wings (FOXC1+). In the second row, maturation of the epithelial identity of the primordium is highlighted by the establishment of apico-basal polarity as hinted by GM130 segregation and basement membrane deposition (Laminin alpha 1 subunit, Lama1). (C) Immunostaining of elongated Gastruloids (120h) against the posterior markers TBra and Cdx2, the endodermal markers FoxA2 and Sox17, the pluripotency marker DPPA4, and the epithelial marker EpCAM to see their localisation within the CDH1+ primordium. A summary expression profile of some of these markers is also provided (gray dotted line indicating the separation between the domain of high TBra but low FoxA2, and that of high FoxA2 and low TBra).Scale bar is always 250um. Marker colocalisation is shown in green and magenta, with double-positive cells appearing white (examples of single-positive and double-positive cells highlighted by single-colour and white arrowheads respectively). $\mathrm{P}=$ posterior of the Gastruloid. 
bioRxiv preprint doi: https://doi.org/10.1101/2020.06.07.138883; this version posted June 10, 2020. The copyright holder for this preprint (which was not certified by peer review) is the author/funder, who has granted bioRxiv a license to display the preprint in perpetuity. It is made available under aCC-BY 4.0 International license.

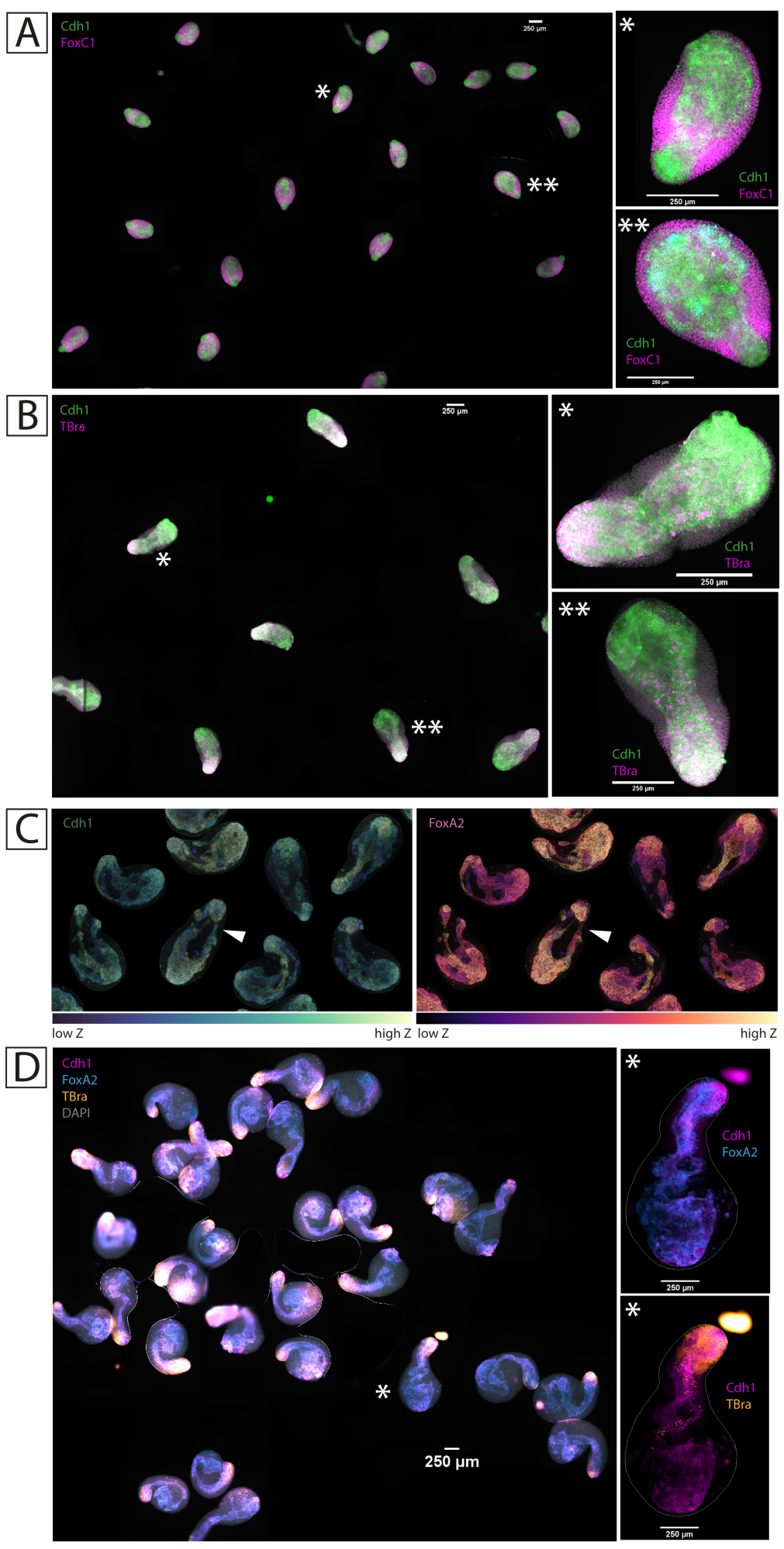

Fig. 5. The CDH1 epithelial primordium is consistently observed in all TBra/Sox1 double reporter Gastruloids ( $\mathrm{n}=\mathbf{9 7 / 9 9 ,} \mathrm{N}=6$ ). Automated scanning of an entire microscope slide of $\mathrm{t}=120 \mathrm{~h}$ Gastruloids, immunostained against $\mathrm{CDH} 1$ and either (A) the pan-mesodermal marker FOXC1, or (B) the posterior epiblast and primitive streak marker TBra. Asterisks indicate samples highlighted at the right of each panel. (C) Depth-coded collection of multiple $\mathrm{t}=120 \mathrm{~h}$ Gastruloids, immunostained for $\mathrm{CDH} 1$ and FOXA2. White arrowhead indicates the sample shown in Figure 4A, right panel. (D) Automated scanning of an entire microscope slide of $t=144 \mathrm{~h}$ Gastruloids, immunostained against CDH1, FOXA2, TBra. The asterisk indicates the sample highlighted at the right. Scale bar is always 250um. 
further characterisation. At the opposite end (anterior), 658 DPPA4+ cells intermingle with FOXA2+ endoderm (Figure 659 4C.8), likely representing a surprising maintenance of 660 pluripotency from the earliest timepoints of Gastruloid 661 differentiation (giving their continuity with DPPA4+ cells 662 at all previous timepoints). On this regard, other groups 663 have interestingly reported the presence of Primordial- 664 Germ-Cell-like cells, marked by DPPA3+, in association 665 with the endodermal component (Veenvliet et al., 2020). 666

Finally, we identify the cell surface protein EpCAM as 668 another marker of the entire primordium, with expression 669 almost completely overlapping that of CDH1 (Figure 4C.9), 670 yet with an apparent enrichment towards the anterior. The 671 expression of EpCAM distinguishes the $(\mathrm{CDH} 1+/) \mathrm{SOX} 17+672$ cells we observe as being indeed endodermal, given that 673 this same marker also characterises endothelial progenitors 674 (which would however be EpCAM-) at around the same 675 developmental timepoints (Choi et al., 2012). Interestingly, 676 EpCAM staining appears enriched in the region of the 677 CDH1 primordium occupied by SOX17+ cells, hinting 678 that combinations of cell-surface markers might drive 679 further sub-sorting of different epithelial combinations 680 within this same $\mathrm{CDH} 1+$ core. Here, the posterior of the 681 primordium would represent a "posterior epiblast"-like, 682 CDH1+/EpCAMlow/TBra+ domain; and the anterior a 683 CDH1+/EpCAMhigh/FOXA2+/Sox17+ "endoderm"-like 684 domain. Further characterisation will highlight whether 685 more refined endoderm identities pattern such domain 686 (Nowotschin et al., 2019b), and whether the absent contri- 687 bution of extraembryonic cells may bias such identities to 688 specific domains.

\section{Discussion}

Cells expressing endodermal markers, and gene expression ${ }_{693}$ patterns consistent with endodermal identities, have been de- ${ }_{694}$ scribed in several previous Gastruloid studies (Beccari et al., ${ }_{695}$ 2018; Pour et al., 2019; Turner et al., 2017; van den Brink ${ }_{696}$ et al., 2020, 2014; Veenvliet et al., 2020). These descriptions ${ }_{697}$ have however often not taken centre stage (with the excep- ${ }_{698}$ tion of (Pour et al., 2019), see later). Of notice is the fact ${ }_{699}$ that FoxA2 and Sox17, indeed classical endoderm markers, 700 are also expressed in other embryonic cell types at the same ${ }_{701}$ developmental stages where the endoderm and the gut tube ${ }_{702}$ are specified. As such, while detecting either of these mark- ${ }_{703}$ ers in the embryo may exclude non-endodermal identities ${ }_{704}$ given the spatial and temporal context of the observation, ${ }_{705}$ the same cannot be said in Gastruloids, where the full extent ${ }_{706}$ of the cell types generated is still under characterisation ${ }_{707}$ (van den Brink et al., 2020), and where temporal alignment ${ }_{708}$ with in vivo developmental stages is uncertain (Beccari et al., ${ }_{709}$ 2018). Accordingly, FOXA2 marks the endoderm just as ${ }_{710}$ much as the neural floorplate and the notochord; TBra marks ${ }_{711}$ posterior hindgut as well as posterior epiblast, notochord, ${ }_{712}$ and neuromesodermal progenitors; and SOX17 marks both ${ }_{713}$ endoderm and endothelial progenitors (Choi et al., 2012). ${ }_{714}$
Regardless, original descriptions of Gastruloids were indeed already describing polarised emergence of endodermal cells expressing both FOXA2 and SOX17 (van den Brink et al., 2014). A compact FOXA2+ domain was thus seen to cluster at the posterior of late stage Gastruloids, with SOX17+/TBracells occupying this very FOXA2+ domain and internalising within epithelial vesicles. We presently cannot reconcile this posteriormost pattern of expression with what we describe here, but we do notice that in those cell lines where the CDH1 primordium does not extend throughout the length of the aggregate it segregates as a compact mass at the posterior (data not shown). The described invagination of $\mathrm{CDH} 1+/ \mathrm{SOX} 17+$ cells may however indeed explain the surface continuity of the posterior $\mathrm{CDH} 1$ primordium that we see in the neck region of some of our Gastruloids.

Even more complete Sox17 patterns have been further described in (Turner et al., 2017), where the use of a reporter highlights the formation of Sox17+ midline, tubular-shaped patterns in elongating gastruloids. The study likens these cells to ventral endodermal cells of the E8.5 mouse embryo. Based on our result, and the extensive SOX17 positivity of the CDH1 primordium we describe here, we would expect the reporter line used in (Turner et al., 2017) to equally give rise to an internal endodermal primordium. Interestingly what we here infer by immunostaining seem to be consistent with the early Sox 17 dynamics described in the paper: Sox $17+$ cells emerging towards the anterior pole of the early Gastruloid and then expanding to occupy a relatively more posterior domain.

SOX17+ cells were also identified in (Beccari et al., 2018), and described as forming tubular structures based on DAPI counterstaining. More importantly, the publication describes gene expression dynamics associated with advanced endoderm maturation: the early upregulation of markers such as Gsc and Cdx2, upregulation of Cer1 during elongation, and expression of posterior foregut markers during later development (Nedn, Sorcs2, Pax9, Pyy, Shh, Krt18). In situ hybridisation patterns for some of these markers are again consistent with the presence of an internal endodermal structure. Still, detailed spatial characterisation is lacking, and the Gastruloid remains framed as a mainly mesenchymal neuromesodermal aggregate. Validation of the maturation of endodermal identities can also be found in the single-cell transcriptomics data generated by (van den Brink et al., 2020), which detect a cluster of cells postulated to represent definitive endoderm as expressing markers Epcam, Col4a1, Sox17.

Most recently, tubular structures populated by FOXA2+ and some SOX17+ and TBra+ cells have also been described in (Veenvliet et al., 2020). Associated with such endoderm-like compartment, the authors also observe Primoridal-Germ-Cells (DPPA3+ cells) which indeed migrate along the gut tube during in vivo development. Our results would seem to suggest that embedding of the 
bioRxiv preprint doi: https://doi.org/10.1101/2020.06.07.138883; this version posted June 10,2020. The copyright holder for this preprint (which was not certified by peer review) is the author/funder, who has granted bioRxiv a license to display the preprint in perpetuity. It is made available under aCC-BY 4.0 International license.

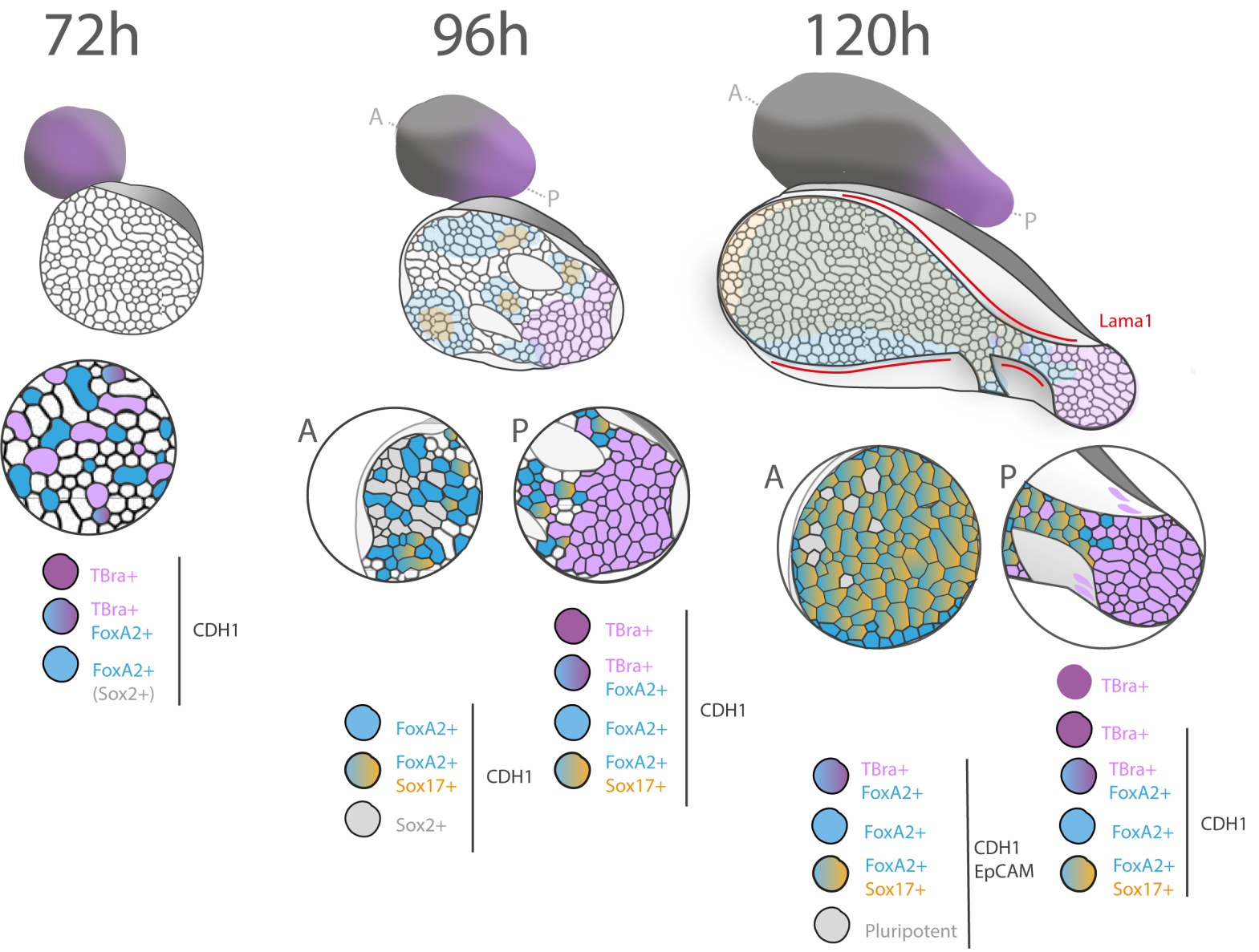

Fig. 6. Endoderm specification and patterning in Gastruloids, a summary. At $72 \mathrm{~h}$ of development, Gastruloids appear to be an epithelioid spheroid with intermingled $\mathrm{TBra}+$ and FOXA2+ $\mathrm{CDH} 1+$ cells. We interpret FOXA2+ cells to also be SOX2+, and this spheroid to represent the posterior epiblast of the early gastrulating mouse embryo, with equally intermingled populations. Between $72 \mathrm{~h}$ and $96 \mathrm{~h}$, Gastruloids undergo widespread Snai1-dependent EMT: mesenchymal types emerge and CDH1 continuity fragments. At the posterior, $\mathrm{CDH} 1+/ \mathrm{TBra}+$ cells define a proximal posterior epiblast compartment and FOXA2+ cells define a distal posterior epiblast region. Anteriorly, the $\mathrm{CDH} 1$ domain remains SOX2+. Within the CDH1+/FOXA2+ domains, SOX17+ cells emerge. In the embryo these cells would be expected to be found in the mesenchymal compartment. At $\mathrm{t}=120 \mathrm{~h}$, the $\mathrm{CDH} 1$ + primordium organises as a maturing epithelium extending along the entire length of the aggregate, enveloped by mesenchymal (mesoderm) cells. FOXA2+ and SOX17+ cells pattern along this primordium, just anteriorly to a posterior-epiblast-like TBra+/CDH1+ domain. EpCAM also seems to mark this very $\mathrm{CDH} 1$ primordium, with an anterior enrichment.

Gastruloid in extracellular matrix components (Veenvliet 734 et al., 2020) is however not necessary to observe endoderm 735 morphogenesis, and that this tissue may actually already 736 organise its own extracellular matrix environment. Unlike ${ }_{737}$ mesoderm thus, which seems to require in vitro ECM cues 738 for productive mesenchymal to epithelial transition (van den 739 Brink et al., 2020; Veenvliet et al., 2020), morphogene- 740 sis of the endoderm appears to be intrinsic to the tissue. ${ }^{741}$

Endoderm emergence in Gastruloids has been atten-743 tively detailed by (Pour et al., 2019), who elegantly used a 744 TBra/Sox 17 double reporter cell line to document expression 745 of these two markers in a temporal sequence consistent 746 with what we describe in this study. Sox17+ cells emerge 747 around 1 day after CHIR exposure, intermingled within 748 a field of TBra+ cells. While the authors also see strong 749 association between Sox17 expression and epitheliality 750 (CDH1 positivity), they do seem to identify a stage where 751 such Sox17+ cells are CDH1-, and indeed favour an interpre- 752 tation that supports a mesendodermal origin of endodermal precursors. In our observations, Gastruloids start in a epithelioid configuration where cells have mesenchymal character but express CDH1, and in which TBra+, FOXA2+, and SOX17+ cells thus all emerge in a $\mathrm{CDH} 1+$ context.

The observations we report here bring centre stage the question of the epithelial character of endodermal precursors, and its link to the fate (both in terms of identity and of location) of these cells (Ferrer-Vaquer et al., 2010; Nowotschin et al., 2019a; Viotti et al., 2014). We expand on previous Gastruloid studies by taking into consideration multiple markers of endodermal identity and documenting their dynamics in relation to one another, and notably by also tracking these cells over time as they undergo morphogenesis. We further also describe sorting of these cells into a complex primordium with maturing epithelial architecture and coarsely patterned domains of gene expression along the AP axis of the organoid. We currently prefer refraining from 
likening this primordium to the early gut tube of the embryo, 810 as more detailed characterisation is underway. At this 811 point, our observations are consistent with a model whereby 812 SOX17+ cells never leave their epithelial environment 813 (initially, the "epiblast"-like domain) and do not need to 814 transition through a mesenchymal state, at least not through 815 classic Snai1-mediated EMT. We speculate that, if SOX17+816 endodermal cells are not being directly specified within the 817 FOXA2+ epiblast, the SOX17+ cells that we incongruously 818 see in this compartment are a result of these cells sticking 819 to neighbours with the same epithelioid character. While in 820 the embryo the isotropic relocalisation of CDH1 associated ${ }_{821}$ with egressed endodermal cells might be compatible with 822 segregation from both epiblast (columnar epithelium) and 823 visceral endoderm (squamous epithelium), and indeed 824 reintegration of Sox $17+$ cells requires re-polarisation, the 825 situation is different in Gastruloids. In our system, FOXA2+ 826 and SOX17+ cells are emerging not in a polarised columnar ${ }_{827}$ epithelium, but in a context that already displays isotropic 828 CDH1 localisation, such that these cells may remain stuck ${ }_{829}$ in their original compartment just by virtue of homotypic 830 interactions. Interestingly, and during preparation of this 831 manuscript, the use of a Cdh1 live reporter by (Hashmi 832 et al., 2020) has shown fragmentation and early sorting 833 dynamics consistent with what we show here by immunos- 834 taining, and the authors explain these sorting dynamics by 835 differences in interfacial tension across cell types. Later ${ }_{836}$ in development we here further observe an expansion of 837 the endodermal population and its internalisation within 838 the core of the Gastruloid as the surface of the aggregate 839 start being populated by an increasing number of mesoder- 840 mal cells. Interestingly, the relative position of different 841 epithelial populations may here again be explained by 842 the expression of different combinations of cell-surface 843 adhesion molecules, a common sorting mechanisms (Toda 844 et al., 2018) that sees here some support from the bi- 845 ased EpCAM distribution within our CDH1 primordium, 846 enriched in the domains occupied by endodermal cells. 847 a maturing epithel throughout late Gastruloid development, contrasting with ${ }_{850}$ the overlaying mesenchymal mesodermal tissues enveloping 851 it, reframes expectations regarding the extent to which fate 852 and morphogenesis can spontaneously arise in vitro. While ${ }_{853}$ gastruloids have traditionally been pictured as aggregates 854 of fates without corresponding organisation, we start to see 855 increasing examples where such missing morphogenesis 856 does occur. As already shown by (Bérenger-Currias et al., 857 2020; van den Brink et al., 2020; Veenvliet et al., 2020) this 858 can be transformatively brought about by the addition of ${ }_{859}$ diluted ECM components or extraembryonic cell types. We here show that differences in epithelial identities between emerging cell types may already be sufficient to generate simple architectures, and that complex epithelia may ${ }^{861}$ spontaneously organise de novo in Gastruloids. Still, the ${ }^{862}$ extent to which the elaborate morphogenesis seen here is ${ }^{863}$ cell-line specific remains to be defined. Current literature ${ }^{864}$ contains examples of Gastruloids from a variety of cell lines and mouse backgrounds that do indeed originate such internal epithelial primordia (Beccari et al., 2018; Rossi et al., 2019) (or where we infer such primordium to be what described (Turner et al., 2017; Veenvliet et al., 2020)), as well as of Gastruloids where such structures do not seem to appear (van den Brink et al., 2020, 2014) and where CDH1+ endodermal tissue segregates to the posteriormost tip of the aggregates instead. We favour the hypothesis that these differences likely correlate to intrinsic cell-line biases in core signalling pathways, and/or on the very degree of epitheliality maintained by these cells by the time they are exposed to CHIR (in turn possibly relating to differences in the 2D culture conditions of these cells). A systematic assessment of the cell-line specific differences in these key parameters remains to be performed. As is the case with the cell line used in this study (Figure 5), we nonetheless expect all Gastruloids generated from a given cell line to consistently produce the same endoderm phenotype in all Gastruloids generated. Regardless, the stratified nature of endodermal and mesodermal tissues we here observe is at least broadly comparable to the configuration of endoderm and mesoderm in the embryo, and it is interesting to speculate that this interfacing may favour the development of more advanced cell fates by reciprocal signalling interactions, as in vivo (Bardot \& Hadjantonakis, 2020; Han et al., 2019).

We will keep this document up to date and share any further characterisation of the $\mathrm{CDH} 1+$ endodermal primordium, and whether FOXA2+ and SOX17+ cells that populate it at 120h further pattern according to the anterior-posterior cues of the aggregate, and to which endodermal organ identities they might give rise to. As per the classical paradigm offered by in vitro developmental models (Shahbazi \& Zernicka-Goetz, 2018), we trust that the extent to which this will match in vivo gut tube development will highlight important developmental principles in both systems. For now, our observations at early stages of Gastruloids development support the existence of endodermal progenitors that do not transition through a TBra+ state and that do not necessarily undergo classic EMT, something debated in the field and for which there is not yet conclusive evidence for in the mouse embryo, but consistent with what seen in other embryonic models (Nowotschin et al., 2019a). Regarding the abnormal epiblast-retention of endodermal cells in Gastruloids, which we explain by the incongruous absence of a starting epithelial architecture in the system, we wonder whether a similar conjuncture would be seen in mutant embryos in which the epiblast does not maintain apico-basal polarity and where epiblast CDH1 may thus be also already isotropically distributed.

\section{Materials and methods}

Cell culture. mESCs ("SBR" Sox1/TBra double reporter cell line described in (Deluz et al., 2016); CRG8 cells of 129P2 background (RRID:CVCL_3987, (Mountford et al., 1994))) were cultured in tissue-culture-treated, nongelatinised, 6well plates, in $10 \%$ Serum Medium with added 
$2 \mathrm{i}$ and LIF. Cells were split every third day, by washing ${ }_{923}$ in PBS-/-, adding Accutase for $\sim 3 \mathrm{~min}$ RT, and collecting 924 the resulting cell suspension in a clean centrifuge tube. 925 The Accutase of the cell suspension was then diluted out 926 1:10 in $10 \%$ Serum Medium, and cells were pelleted by centrifuging $200 \mathrm{xg}, 4 \mathrm{~min}, 4^{\circ} \mathrm{C}$. After aspirating out the ${ }^{927}$ supernatant, the pellet was resuspended in $1 \mathrm{~mL} 10 \%$ Serum ${ }^{928}$ Medium to a single cell suspension, and cell density was ${ }^{929}$ counted with a haemocytometer. Around 65000-75000 cells 930 were transferred to a new well with $2 \mathrm{~mL}$ pre-equilibrated 931 10\%Serum Medium (6750-7800 cells/cm2). Cells were $9 з 2$ then left in a humidified incubator, $37^{\circ} \mathrm{C}, 5 \% \mathrm{CO}_{2}$, until 93 use or until further splitting 3 days later. NOTE: In most 934 cases, splitting was coupled to Gastruloid generation. In ${ }^{3} 5$ those cases, the cell pellet was resuspended in N2B27 936 rather than $10 \%$ Serum Medium $+2 \mathrm{i}$ and LIF. A com- ${ }^{937}$ plete, step-by-step, protocol is available at:https:// 9з8 dx.doi.org/10.17504/protocols.io.7xbhpin 939 Recipes:

10\% Serum Medium: 86.8\% DMEM, high glucose, with Glu- 941 taMAX (L-Alanyl-Glutamine, final concentration: $3.45 \mathrm{mM}$ ), 942 $10 \%$ ES-grade Foetal Bovine Serum, 100U/mL Penicillin, 943 $100 \mathrm{ug} / \mathrm{mL}$ Streptomycin, $0.1 \mathrm{mM}$ Non Essential Amino 944 Acids, $1 \mathrm{mM}$ Sodium Pyruvate, $0.1 \mathrm{mM}$ beta-mercaptoethanol 945 10\% Serum Medium + 2i and LIF: add 3uM CHIR99021, 946 1uM PD0325901, and 100u/mL LIF.

Gastruloid generation. mESCs were washed in PBS-/-, 949 detached from adherent culture with Accutase ( $\sim 3 \mathrm{~min}, \mathrm{RT})$, ${ }_{950}$ and collected in a centrifuge tube. The Accutase in the ${ }_{951}$ cell suspension was then diluted out 1:10 in 10\% Serum $_{952}$ Medium, and cells were pelleted by centrifuging 200xg, ${ }_{953}$ $4 \mathrm{~min}, 4 \mathrm{C}$. The supernatant was removed, and the pellet was ${ }_{954}$ washed by resuspension in $10 \mathrm{~mL}$ PBS-/-. Cells were re- 955 pelleted by centrifuging $200 \mathrm{xg}, 4 \mathrm{~min}, 4 \mathrm{C}$ and washed once ${ }_{956}$ more in $10 \mathrm{~mL}$ fresh PBS-/-. After re-pelleting once more (200xg, 4min, 4C), the pellet was dissociated as a single-cell 957 suspension in $1 \mathrm{~mL} \mathrm{N2B27} \mathrm{Medium.} \mathrm{Cells} \mathrm{were} \mathrm{counted} 958$ with a haemocytometer, and, for each plate of Gastruloids 959 made, 37500 cells were transferred to $5 \mathrm{~mL}$ fresh N2B27 960 (7.5 cells/uL final concentration). The cell suspension was 961 distributed as $40 \mathrm{uL}$ droplets (=300 cells/droplet) in wells of ${ }_{962}$ a U-bottomed, low-adhesion, 96 well plate, and the plates 963 were left for $120 \mathrm{~h}$ in a humidified incubator, $5 \% \mathrm{CO}_{2}, 37^{\circ} \mathrm{C} .964$ At $48 \mathrm{~h}$ after plating, 150uL of 3uM CHIR99021 N2B27 965 were added to each well, and this solution was substituted 966 with fresh N2B27 (no CHIR) every 24h after that. A step- 967 by-step detailed protocol is available at: https://dx. 968 doi.org/10.17504/protocols.io.9j5h4q6 Recipes: $\quad 10 \%$ Serum Medium: $86.8 \%$ DMEM, 970 high glucose, with $3.97 \mathrm{mM}$ GlutaMAX (L-Alanyl-971 Glutamine, final concentration: $3.45 \mathrm{mM}), \quad 10 \% 972$ ES-grade FBS, 100U/mL Penicillin , $100 \mathrm{ug} / \mathrm{mL}_{973}$ Streptomycin, $0.1 \mathrm{mM}$ Non Essential Amino Acids, 974 $1 \mathrm{mM}$ Sodium Pyruvate, $1 \mathrm{mM}$ beta-mercaptoethanol. 975 N2B27: 47.4\% Neurobasal Medium, 47.4\% DMEM/F- 976 12 , with $2.50 \mathrm{mM}$ GlutaMAX (L-Alanyl-Glutamine, final 977 concentration: $1.18 \mathrm{mM}), 1 \mathrm{mM}$ GlutaMAX Supplement 978 (total concentration: 2.18mM), 100U/mL Penicillin , $100 \mathrm{ug} / \mathrm{mL}$ Streptomycin, 0.1mM Non Essential Amino Acids, $1 \mathrm{mM}$ Sodium Pyruvate, $1 \mathrm{mM}$ beta-mercaptoethanol, $1 \%$ B27Supplement, serum-free, 0.5\% N-2 Supplement.

Gastruloid immunostaining. Gastruloids were collected at every given timepoint, washed in PBS-/-, and fixed in $4 \%$ PFA in PBS-/-, for $2 \mathrm{~h}, 4 \mathrm{C}$, on a low-speed orbital shaker; or $45 \mathrm{~min}$, RT, static. Gastruloids were then washed in PBS+FT (PBS-/-, 10\% ES-grade Foetal Bovine Serum, $0.2 \%$ Triton-X100), and blocked and permeabilised in PBS+FT for $1 \mathrm{~h}$, RT, static. Primary antibody solutions were then prepared in PBS+FT, with $2 \mathrm{ug} / \mathrm{mL}$ DAPI. Samples were stained overnight, $4 \mathrm{C}$, on a low-speed orbital shaker. Similarly, secondary antibody solutions were prepared in PBS+FT, 2ug/mL DAPI, and samples were stained overnight, 4C, on a low-speed orbital shaker. Gastruloids were mounted in Fluoromount-G mounting medium (no spacers), and slides kept at 4C long term. All antibody solutions were washed away after incubation by washes in PBS+FT. A detailed, step-by-step protocol is available at: https://dx.doi.org/10.17504/protocols. io.7tzhnp6. Secondary antibodies used were all from Thermo Fisher Scientific: donkey anti-mouse IgG Alexa 647 (CAT\#A-31571, RRID:AB_162542); donkey anti-rabbit IgG Alexa 488 (CAT\#A-21206, RRID:AB_2535792), Alexa 568 (CAT\# A-10042, RRID:AB_2534017), or Alexa 647 (CAT\# A-31573, RRID:AB_2536183); donkey anti-rat IgG Alexa 488 (CAT\#A-21208, RRID:AB_2535794), goat anti-rat Alexa 568 (CAT\#A-11077, RRID:AB_2534121), or Alexa 647 (CAT\#A-21247, RRID:AB_141778); donkey anti-goat IgG Alexa 488 (CAT\#A-11055, RRID:AB_2534102), or Alexa 568 (CAT\#RRID:AB_2534104). Details about the primary antibodies used are provided as a supplementary .csv file.

Gastruloid imaging and image processing. Brightfield images of Gastruloids were taken on either a Nikon Ti inverted spinning-disk microscope (for the series in Figure 2A), or an Olympus CellR inverted widefield microscope (for the image on Figure 4B, UPLAN S APO 10x/0.40 air objective, CCD Grayscale Hamamatsu ORCA ER B7W Camera; Olympus XCellence software for data capture). Both microscope setups had $\mathrm{CO} 2$ and temperature control $\left(37^{\circ} \mathrm{C}\right.$ and $\left.5 \% \mathrm{CO}_{2}\right)$. Immunostained Gastruloids were imaged on a Zeiss LSM700 inverted confocal microscope (Plan-Apochromat 20x/0.80 air objective, motorized stage, LED Lumencor SOLA Illumination, CCD Grayscale Axiocam MRm (B/W) Camera; ZEN 2009 software for data capture) or on a Zeiss LSM780 inverted confocal microscope (for the CDH1 projection in Figure 4A, PlanApochromat 20x/0.80 air objective, motorized stage). Images were opened, stitched, and processed for publication (LUT assignment, channel display, min and max intensity thresholding based on no-primary control) using the Fiji ImageJ distribution (Rueden et al., 2017; Schindelin et al., 2012), and the "Grid/Collection Stitch- 
bioRxiv preprint doi: https://doi.org/10.1101/2020.06.07.138883; this version posted June 10, 2020. The copyright holder for this preprint (which was not certified by peer review) is the author/funder, who has granted bioRxiv a license to display the preprint in perpetuity. It is made available under aCC-BY 4.0 International license.

ing" plugin therein (Preibisch et al., 2009). The depth-1038 coded reconstruction in Figure 4A was generated using the "Temporal-Color Code" (https://imagej.net/1030 Temporal-Color_Code) function. The blue, orange, 1041 and purple LUTs used throughout the figures were $\mathrm{de}_{-1043}^{1042}$ signed by Christophe Leterrier (https : / / github. com/1044 cleterrier/ChrisLUTs, "BOP" palette).

Quantification of AP patterning. Batch quantification of ${ }_{1051}^{1050}$ immunostaining signal intensity along the AP axis of the 1052 Gastruloids was performed through a custom processing $g_{1054}^{1053}$ pipeline available as a Jupyter notebook at https:// doi.1055 org/10.5281/zenodo.3884238 and outlined as fol- ${ }^{1056}$ lows (step-by-step walk through provided in the notebook 1058 itself). The pipeline takes two inputs: i) the multichannel $1_{1060}^{1059}$ raw image resulting from the scan of an entire microscope 1061 slide of immunostained and mounted Gastruloids (here ac- ${ }^{1062}$ quired on a GE Healthcare IN Cell Analyzer 2200 automated1064 microscope) and ii) hand-traced line coordinates defining the $\mathrm{e}_{1066}^{1065}$ central axis of each Gastruloid on the slide (starting from the1067 posterior). At early timepoints where the posterior of the ${ }_{1069}^{1068}$ Gastruloid is not distinguishable morphologically, the area1070 of TBra polarisation is to be used instead. The script then ${ }_{1072}^{1071}$ subdivides each interval of the line ROI provided into $n$ finer1073 intervals of equal length (thus avoiding to have to manually $y_{1075}^{1074}$ draw a line with high number of points; here $n=10$ ), and for 1076 each point along the line it defines a non-overlapping polygon ${ }_{1078}^{1077}$ mask covering an area of thickness $N$ (here $N=500 \mathrm{px}$ ) across 1079 the line and whose lateral edges are orthogonal to the line it ${ }^{1080}$ self at each side of the point. Having computed the mask, the1082 script then assigns the total signal intensity recovered in the $\mathrm{e}_{1084}^{1083}$ area to the point of the line ROI around which the polygon 1085 was constructed, thus effectively assigning signal intensities ${ }_{1087}^{1086}$ to points that can be ordered along an $\mathrm{x}$-axis. These raw val-1088 ues are then normalised by the number of cells in the area ${ }_{1090}^{1089}$ (using the DAPI nuclear intensity as a proxy) and both posi-1091 tion along the length of the Gastruloid and signal intensity $\operatorname{are}_{1093}^{1092}$ normalised to the absolute length of the Gastruloid and to the 1094 maximal DAPI-normalised intensity value. The script out $-_{1096}^{1095}$ puts lineplots and scatterplots for each Gastruloid analysed,1097 summary lineplots and scatterplots with collated data of all ${ }^{1098}$ gastruloids analysed, and the tabulated raw data for re-use. ${ }_{1100}$

\section{ACKNOWLEDGEMENTS}

We would like to acknowledge the work and support provided by the staff of many ${ }^{1104}$ of the EPFL core facilities: notably, the staff of the Biolmaging and Optics Platform ${ }^{1105}$ (BIOP), the staff of the Biomolecular Screening Facility (BSF), and the staff of the ${ }^{1106}$ Histology Core Facility. We also want to acknowledge the work of the entire staff of 107 the Glassware Washing facility (Laverie), as well as all of EPFL cleaning staff. This'108 work would have not been possible without their contribution. The pipeline designed1109 to quantify AP patterning in Gastruloids was constructed in collaboration with Paull110 Gerald Layague Sanchez and Arianne Bercowsky-Rama, whom we sincerely thank. ${ }^{1111}$ We would also like to acknowledge our lab manager Stephanie Boy-Rottinger for her1112 heartfelt support throughout. In addition, we are thankful to Ricardo Henriques for ${ }^{1113}$ kindly sharing the template that was used to format this preprint. We also thank $k^{1114}$ Alfonso Martinez Arias for valuable feedback on the first uploaded bioRxiv version ${ }^{1115}$ of this manuscript. This research was funded by a Sinergia grant (CRSII5_189956)1116 from the Swiss National Science Foundation and Ecole Polytechnique Fédérale de ${ }^{1117}$ Lausanne (EPFL).

\section{COMPETING FINANCIAL INTERESTS}

The authors declare no competing financial interests.

\section{Bibliography}

Allman, G. J. (1854). On the anatomy and physiology of Cordylophora a contribution to our knowledge of the tubularian zoophytes. Abstracts of the Papers Communicated to the Royal Society of London, 6, 319-321.

URL https://doi.org/10.1098\%2Frspl.1850.0118

Arnold, S. J., \& Robertson, E. J. (2009). Making a commitment: cell lineage allocation and axis patterning in the early mouse embryo. Nature Reviews Molecular Cell Biology, 10(2), 91103.

URL https: //doi.org/10.1038\%2Fnrm2618

Baillie-Johnson, P., van den Brink, S. C., Balayo, T., Turner, D. A., \& Arias, A. M. (2015). Generation of Aggregates of Mouse Embryonic Stem Cells that Show Symmetry Breaking Polarization and Emergent Collective Behaviour emIn Vitro/em. Journal of Visualized Experiments, (105).

URL https://doi.org/10.3791\%2F53252

Bardot, E., Calderon, D., Santoriello, F., Han, S., Cheung, K., Jadhav, B., Burtscher, I., Artap, S., Jain, R., Epstein, J., Lickert, H., Gouon-Evans, V., Sharp, A. J., \& Dubois, N. C. (2017). Foxa2 identifies a cardiac progenitor population with ventricular differentiation potential. $\mathrm{Na}$ ture Communications, $8(1)$.

URL https://doi.org/10.1038\%2Fncomms14428

Bardot, E. S., \& Hadjantonakis, A.-K. (2020). Mouse gastrulation: Coordination of tissue patterning specification and diversification of cell fate. Mechanisms of Development, (p. 103617). URL https://doi.org/10.1016\%2Fj.mod.2020.103617

Beccari, L., Moris, N., Girgin, M., Turner, D. A., Baillie-Johnson, P., Cossy, A.-C., Lutolf, M. P., Duboule, D., \& Arias, A. M. (2018). Multi-axial self-organization properties of mouse embryonic stem cells into gastruloids. Nature, 562(7726), 272-276.

URL https://doi.org/10.1038\%2Fs41586-018-0578-0

Beck, F., Erler, T., Russell, A., \& James, R. (1995). Expression of Cdx-2 in the mouse embryo and placenta: Possible role in patterning of the extra-embryonic membranes. Developmental Dynamics, 204(3), 219-227.

URL https://doi.org/10.1002\%2Faja.1002040302

Bérenger-Currias, N. M. L. P., Mircea, M., Adegeest, E., van den Berg, P. R., Feliksik, M., Hochane, M., Idema, T., Tans, S. J., \& Semrau, S. (2020). Early neurulation recapitulated in assemblies of embryonic and extraembryonic cells.

URL https://doi.org/10.110122020.02.13.947655

Burtscher, I., \& Lickert, H. (2009). Foxa2 regulates polarity and epithelialization in the endoderm germ layer of the mouse embryo. Development, 136(6), 1029-1038.

URL https://doi.org/10.1242\%2Fdev. 028415

Cano, A., Pérez-Moreno, M. A., Rodrigo, I., Locascio, A., Blanco, M. J., del Barrio, M. G., Portillo, F., \& Nieto, M. A. (2000). The transcription factor Snail controls epithelial-mesenchymal transitions by repressing E-cadherin expression. Nature Cell Biology, 2(2), 76-83. URL https://doi.org/10.1038\%2F35000025

Carlson, B. M. (2014). Digestive and Respiratory Systems and Body Cavities. In Human Embryology and Developmental Biology, (pp. 335-375). Elsevier. URL https://doi.org/10.1016\%2Fb978-1-4557-2794-0.00015-2

Carver, E. A., Jiang, R., Lan, Y., Oram, K. F., \& Gridley, T. (2001). The Mouse Snail Gene Encodes a Key Regulator of the Epithelial-Mesenchymal Transition. Molecular and Cellular Biology, 21(23), 8184-8188.

URL https://doi.org/10.1128\%2Fmcb.21.23.8184-8188.2001

Cermola, F., D'Aniello, C., Tatè, R., De Cesare, D., Martinez-Arias, A., Minchiotti, G., \& Patriarca, E. J. (2019). Gastruloid development competence discriminates different states of pluripotency between naïve and primed. bioRxiv, (p. 664920).

Chazaud, C., Yamanaka, Y., Pawson, T., \& Rossant, J. (2006). Early Lineage Segregation between Epiblast and Primitive Endoderm in Mouse Blastocysts through the Grb2-MAPK Pathway. Developmental Cell, 10(5), 615-624.

URL https://doi.org/10.1016\%2Fj.devcel.2006.02.020

Choi, E., Kraus, M. R.-C., Lemaire, L. A., Yoshimoto, M., Vemula, S., Potter, L. A., Manduchi, E., Stoeckert, C. J., Grapin-Botton, A., \& Magnuson, M. A. (2012). Dual Lineage-Specific Expression of Sox17 During Mouse Embryogenesis. STEM CELLS, 30(10), 2297-2308. URL https://doi.org/10.1002\%2Estem.1192

Davies, J. (2017). Using synthetic biology to explore principles of development. Development, 144(7), 1146-1158.

URL https://doi.org/10.1242\%2Fdev.144196

Deluz, C., Friman, E. T., Strebinger, D., Benke, A., Raccaud, M., Callegari, A., Leleu, M., Manley, S., \& Suter, D. M. (2016). A role for mitotic bookmarking of SOX2 in pluripotency and differentiation. Genes \& Development, 30(22), 2538-2550.

URL https://doi.org/10.1101\%2Fgad.289256.116

Dufort, D., Schwartz, L., Harpal, K., \& Rossant, J. (1998). The transcription factor HNF3beta is required in visceral endoderm for normal primitive streak morphogenesis. Development, 125 , 3015-25.

Ferrer-Vaquer, A., Viotti, M., \& Hadjantonakis, A.-K. (2010). Transitions between epithelial and mesenchymal states and the morphogenesis of the early mouse embryo. Cell adhesion \& migration, 4(3), 447-457.

Hamidi, S., Nakaya, Y., Nagai, H., Alev, C., Kasukawa, T., Chhabra, S., Lee, R., Niwa, H., Warmflash, A., Shibata, T., \& Sheng, G. (2020). Mesenchymal-epithelial transition regulates initiation of pluripotency exit before gastrulation. Development, 147(3), dev184960. URL https://doi.org/10.1242\%2Fdev.184960

Han, L., Chaturvedi, P., Kishimoto, K., Koike, H., Nasr, T., Iwasawa, K., Giesbrecht, K., Witcher, P. C., Eicher, A., Haines, L., Lee, Y., Shannon, J. M., Morimoto, M., Wells, J. M., Takebe, T., \& Zorn, A. M. (2019). Single cell transcriptomics reveals a signaling roadmap coordinating endoderm and mesoderm diversification during foregut organogenesis. URL https://doi.org/10.1101\%2F756825

Hashimoto, K., \& Nakatsuji, N. (1989). Formation of the Primitive Streak and Mesoderm Cells in Mouse Embryos-Detailed Scanning Electron Microscopical Study. (primitive streak/cell migration/extracellular matrix/mouse gastrulation/scanning electron microscopy). Development Growth and Differentiation, 31(3), 209-218. 
bioRxiv preprint doi: https://doi.org/10.1101/2020.06.07.138883; this version posted June 10, 2020. The copyright holder for this preprint (which was not certified by peer review) is the author/funder, who has granted bioRxiv a license to display the preprint in perpetuity. It is made available under aCC-BY 4.0 International license.

URL https://doi.org/10.1111\%2Fj.1440-169x.1989.00209.x URL https://doi.org/10.1101\%2F2020.05.21.105551

Herrmann, B. (1991). Expression pattern of the Brachyury gene in whole-mount TWis/TWis mu-1213 tant embryos. Development, 113, 913-7.

Hiramatsu, R., Matsuoka, T., Kimura-Yoshida, C., Han, S.-W., Mochida, K., Adachi, T., Takayama,1215 S., \& Matsuo, I. (2013). External Mechanical Cues Trigger the Establishment of the Anterior-1216 Posterior Axis in Early Mouse Embryos. Developmental Cell, 27(2), 131-144. URL https://doi.org/10.1016\%2Fj.devcel.2013.09.026

anai-Azuma, M., Kanai, Y., Gad, J., Tajima, Y., Taya, C., Kurohmaru, M., Sanai, Y., Yonekawa, H.,1219 Yazaki, K., Tam, P., \& Hayashi, Y. (2002). Depletion of definitive gut endoderm in Sox17-null1220 mutant mice. Development, 129, 2367-79.

Kubo, A., Shinozaki, K., Shannon, J., Kouskoff, V., Kennedy, M., Woo, S., Fehling, H., \& Keller, G.1222 (2004). Development of definitive endoderm from embryonic stem cells in culture. Develop-1223 ment, 131, 1651-62.

Kwon, G. S., Viotti, M., \& Hadjantonakis, A.-K. (2008). The Endoderm of the Mouse Embryo1225 Arises by Dynamic Widespread Intercalation of Embryonic and Extraembryonic Lineages.122 Developmental Cell, 15(4), 509-520.

URL https: //doi.org/10.1016\%2Fj.devcel.2008.07.017

Lee, J. D., Silva-Gagliardi, N. F., Tepass, U., McGlade, C. J., \& Anderson, K. V. (2007). The122 ferm protein epb4. 115 is required for organization of the neural plate and for the epithelial-1230 mesenchymal transition at the primitive streak of the mouse embryo. Development, 134(11).123

Lewis, S. L., \& Tam, P. P. (2006). Definitive endoderm of the mouse embryo: Formation cell fates,1232 and morphogenetic function. Developmental Dynamics, 235(9), 2315-2329. URL https://doi.org/10.1002\%2Fdvdy.20846

Matsuo, I \& Hiramatsu, R (2017). Mechanical perspectives on the anterior-posterior axis polar-1235 ization of mouse implanted embryos. Mechanisms of Development, 144, 62-70. URL https://doi.org/10.1016\%2Fj.mod.2016.09.002

McGrath, P., \& Wells, J. (2015). SnapShot: GI tract development. Cell, 161, 176-176.e1. Monaghan, A., Kaestner, K., Grau, E., \& Schütz, G. (1993). Postimplantation expression patterns1239 indicate a role for the mouse forkhead/HNF-3 alpha, beta and gamma genes in determination1240 of the definitive endoderm, chordamesoderm and neuroectoderm. Development, 119, 567-124 78 .

Mountford, P., Zevnik, B., Duwel, A., Nichols, J., Li, M., Dani, C., Robertson, M., Chambers, I.,124 \& Smith, A. (1994). Dicistronic targeting constructs: reporters and modifiers of mammalian1244 gene expression. Proceedings of the National Academy of Sciences, 91(10), 4303-4307. 1245 URL https://doi.org/10.1073\%2Fpnas.91.10.4303

Nowotschin, S., Hadjantonakis, A.-K., \& Campbell, K. (2019a). The endoderm: a divergent celli247 lineage with many commonalities. Development, 146(11), dev150920.

URL https://doi.org/10.1242\%2Fdev.150920 Gardner, R., Boutet, S. C., Church, D. M., Hoodless, P. A., Hadjantonakis, A.-K., \& Pe'er,125 D. (2019b). The emergent landscape of the mouse gut endoderm at single-cell resolution.1252 Nature, 569(7756), 361-367.

URL https://doi.org/10.1038\%2Fs41586-019-1127-1

Oppenheimer, J. M. (1940). The Non-Specificity of the Germ-Layers. The Quarterly Review ofi255 Biology, 15(1), 1-27. URL https://doi.org/10.1086\%2F394599

Pander, C. H. (1817). Dissertatio inauguralis sistens historiam metamorphoseos quam ovum incubatum prioribus quinque diebus subit.. Typis Nitribitt,. URL https://doi.org/10.5962\%2Fbhl.title.48488

Peter, l., \& Davidson, E. (2010). The endoderm gene regulatory network in sea urchin embryos up to mid-blastula stage. Dev Biol, 340, 188-99.

Pfendler, K., Catuar, C., Meneses, J., \& Pedersen, R. (2005). Overexpression of Nodal promotes differentiation of mouse embryonic stem cells into mesoderm and endoderm at the expense of neuroectoderm formation. Stem Cells Dev, 14, 162-72.

Pour, M., Kumar, A. S., Walther, M., Wittler, L., Meissner, A., \& Nachman, I. (2019). Emergence and patterning dynamics of mouse definitive endoderm.

URL https://doi.org/10.11012F728642

Preibisch, S., Saalfeld, S., \& Tomancak, P. (2009). Globally optimal stitching of tiled 3D microscopic image acquisitions. Bioinformatics, 25(11), 1463-1465. URL https://doi.org/10.1093\%2Fbioinformatics\%2Fbtp184

Rossi, G., Boni, A., Guiet, R., Girgin, M., Kelly, R. G., \& Lutolf, M. P. (2019). Embryonic organoids recapitulate early heart organogenesis. URL https://doi.org/10.1101\%2F802181

Rueden, C., Schindelin, J., Hiner, M., DeZonia, B., Walter, A., Arena, E., \& Eliceiri, K. (2017). ImageJ2: ImageJ for the next generation of scientific image data. BMC Bioinformatics, 18, 529.

Sasaki, H., \& Hogan, B. (1993). Differential expression of multiple fork head related genes during gastrulation and axial pattern formation in the mouse embryo. Development, 118, 47-59.

Saykali, B., Mathiah, N., Nahaboo, W., Racu, M.-L., Hammou, L., Defrance, M., \& Migeotte, I. (2019). Distinct mesoderm migration phenotypes in extra-embryonic and embryonic regions of the early mouse embryo. Elife, 8, e42434.

Schindelin, J., Arganda-Carreras, I., Frise, E., Kaynig, V., Longair, M., Pietzsch, T., Preibisch, S., Rueden, C., Saalfeld, S., Schmid, B., Tinevez, J.-Y., White, D. J., Hartenstein, V., Eliceiri, K., Tomancak, P., \& Cardona, A. (2012). Fiji: an open-source platform for biological-image analysis. Nature Methods, 9(7), 676-682.

URL https://doi.org/10.1038\%2Fnmeth.2019

Shahbazi, M. N., \& Zernicka-Goetz, M. (2018). Deconstructing and reconstructing the mouse and human early embryo. Nature Cell Biology, 20(8), 878-887.

URL https://doi.org/10.10382Fs41556-018-0144-x

Smith, L. (1985). Embryonic axis orientation in the mouse and its correlation with blastocyst relationships to the uterus. II. Relationships from $41 / 4$ to $91 / 2$ days. J Embryol Exp Morphol, $89,15-35$.

Stower, M., \& Srinivas, S. (2018). The Head's Tale: Anterior-Posterior Axis Formation in the
Mouse Embryo. Curr Top Dev Biol, 128, 365-390.

Sulston, J., Schierenberg, E., White, J., \& Thomson, J. (1983). The embryonic cell lineage of the nematode Caenorhabditis elegans. Dev Biol, 100, 64-119.

Tada, S., Era, T., Furusawa, C., Sakurai, H., Nishikawa, S., Kinoshita, M., Nakao, K., Chiba, T., \& Nishikawa, S. (2005). Characterization of mesendoderm: a diverging point of the definitive endoderm and mesoderm in embryonic stem cell differentiation culture. Development, 132, 4363-74.

Takaoka, K., \& Hamada, H. (2012). Cell fate decisions and axis determination in the early mouse embryo. Development, 139, 3-14

Tam, P., \& Behringer, R. (1997). Mouse gastrulation: the formation of a mammalian body plan. Mech Dev, 68, 3-25.

Tam, P. P. L., \& Loebel, D. A. F. (2007). Gene function in mouse embryogenesis: get set for gastrulation. Nature Reviews Genetics, 8(5), 368-381. URL https://doi.org/10.1038\%2Fnrg2084

Toda, S., Blauch, L. R., Tang, S. K. Y., Morsut, L., \& Lim, W. A. (2018). Programming selforganizing multicellular structures with synthetic cell-cell signaling. Science, (p. eaat0271). URL https://doi.org/10.1126\%2Fscience.aat0271

Turner, D., Baillie-Johnson, P., \& Martinez, A. A. (2016). Organoids and the genetically encoded self-assembly of embryonic stem cells. Bioessays, 38, 181-91.

Turner, D. A., Girgin, M., Alonso-Crisostomo, L., Trivedi, V., Baillie-Johnson, P., Glodowski, C. R., Hayward, P. C., Collignon, J., Gustavsen, C., Serup, P., Steventon, B., Lutolf, M. P., \& Arias, A. M. (2017). Anteroposterior polarity and elongation in the absence of extra-embryonic tissues and of spatially localised signalling in gastruloids: mammalian embryonic organoids. Development, 144(21), 3894-3906.

URL https://doi.org/10.1242\%2Fdev.150391

van den Brink, S. C., Alemany, A., van Batenburg, V., Moris, N., Blotenburg, M., Vivié, J., BaillieJohnson, P., Nichols, J., Sonnen, K. F., Arias, A. M., \& van Oudenaarden, A. (2020). Singlecell and spatial transcriptomics reveal somitogenesis in gastruloids. Nature. URL https://doi.org/10.1038\%2Fs41586-020-2024-3

van den Brink, S. C., Baillie-Johnson, P., Balayo, T., Hadjantonakis, A.-K., Nowotschin, S., Turner, D. A., \& Arias, A. M. (2014). Symmetry breaking germ layer specification and axial organisation in aggregates of mouse embryonic stem cells. Development, 141(22), 4231-4242. URL https://doi.org/10.1242\%2Fdev.113001

Veenvliet, J. V., Bolondi, A., Kretzmer, H., Haut, L., Scholze-Wittler, M., Schifferl, D., Koch, F., Pustet, M., Heimann, S., Buschow, R., Wittler, L., Timmermann, B., Meissner, A., \& Herrmann, B. G. (2020). Mouse embryonic stem cells self-organize into trunk-like structures with neural tube and somites.

URL https://doi.org/10.1101\%2F2020.03.04.974949

Viotti, M., Nowotschin, S., \& Hadjantonakis, A.-K. (2014). SOX17 links gut endoderm morphogenesis and germ layer segregation. Nature Cell Biology, 16(12), 1146-1156. URL https://doi.org/10.1038\%2Fncb3070

Williams, M., Burdsal, C., Periasamy, A., Lewandoski, M., \& Sutherland, A. (2011). Mouse primitive streak forms in situ by initiation of epithelial to mesenchymal transition without migration of a cell population. Developmental Dynamics, 241(2), 270-283. URL https://doi.org/10.1002\%2Fdvdy.23711

Yamanaka, Y., Tamplin, O. J., Beckers, A., Gossler, A., \& Rossant, J. (2007). Live Imaging and Genetic Analysis of Mouse Notochord Formation Reveals Regional Morphogenetic Mechanisms. Developmental Cell, 13(6), 884-896.

URL https://doi.org/10.1016\%2Fj.devcel.2007.10.016 